Journal of Business \& Economic

Statistics

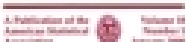

\section{Journal of Business \& Economic Statistics}

ISSN: 0735-0015 (Print) 1537-2707 (Online) Journal homepage: https://www.tandfonline.com/loi/ubes20

\title{
The Finite Sample Performance of Estimators for Mediation Analysis Under Sequential Conditional Independence
}

\section{Martin Huber, Michael Lechner \& Giovanni Mellace}

To cite this article: Martin Huber, Michael Lechner \& Giovanni Mellace (2016) The Finite Sample Performance of Estimators for Mediation Analysis Under Sequential Conditional Independence, Journal of Business \& Economic Statistics, 34:1, 139-160, DOI: 10.1080/07350015.2015.1017644

To link to this article: https://doi.org/10.1080/07350015.2015.1017644

Accepted author version posted online: 11

Mar 2015.

Published online: 20 Jan 2016.

Submit your article to this journal

山 Article views: 549

Q View related articles 5

View Crossmark data $־$

Citing articles: 4 View citing articles ¿ 


\title{
The Finite Sample Performance of Estimators for Mediation Analysis Under Sequential Conditional Independence
}

\author{
Martin Huber \\ Department of Economics, University of Fribourg, Bd. de Pérolles 90, $\mathrm{CH}-1700$ Fribourg, Switzerland \\ (martin.huber@unifr.ch) \\ Michael LECHNER \\ Swiss Institute for Empirical Economic Research, University of St. Gallen, Varnbüelstrasse 14, $\mathrm{CH}-9000$, \\ St. Gallen, Switzerland (michael.lechner@unisg.ch) \\ Giovanni Mellace \\ Department of Business and Economics, University of Southern Denmark, Campusvej 55, DK-5230 \\ Odense M, Denmark (giome@sam.sdu.dk)
}

\begin{abstract}
Using a comprehensive simulation study based on empirical data, this article investigates the finite sample properties of different classes of parametric and semiparametric estimators of (natural) direct and indirect causal effects used in mediation analysis under sequential conditional independence assumptions. The estimators are based on regression, inverse probability weighting, and combinations thereof. Our simulation design uses a large population of Swiss jobseekers and considers variations of several features of the datagenerating process (DGP) and the implementation of the estimators that are of practical relevance. We find that no estimator performs uniformly best (in terms of root mean squared error) in all simulations. Overall, so-called "g-computation" dominates. However, differences between estimators are often (but not always) minor in the various setups and the relative performance of the methods often (but not always) varies with the features of the DGP.
\end{abstract}

KEY WORDS: Causal channels; Causal mechanisms; Direct effects; Empirical Monte Carlo study; Indirect effects; Simulation.

\section{INTRODUCTION}

A vast empirical literature in statistical and social sciences is concerned with the estimation of causal effects, both in randomized experiments and in observational studies. However, in many studies not only the causal effect per se appears interesting, but also the causal mechanisms through which it operates. Or, as Gelman and Imbens (2013) argued, very often not only the effects of causes appear relevant, but also the causes of effects. For example, when assessing the employment or earnings effects of an active labor market program, policy makers might want to know to which extent the total impact comes from increased search effort, human capital, or other mediators that are themselves affected by the program. It is therefore no surprise that the analysis of the causal mechanisms through which an initial treatment variable affects an outcome of interest, also referred to as mediation analysis, has become more relevant in empirical work across disciplines such as economics, epidemiology, political science, and statistics. Identification requires controlling for the potential endogeneity of the treatment and the intermediate variables through which the causal mechanisms operate (i.e., through which the treatment ultimately affects the outcome of interest), the so-called mediators. Under particular assumptions, the (total) causal effect may then be disentangled in an indirect component related to one or several mediators and a direct effect, which includes all remaining causal channels not covered by the mediators of interest.

The majority of studies in mediation analysis assumes that all potential confounders jointly affecting the treatment, the mediator, and the outcome (and thus potentially causing treatment and/or mediator endogeneity) are observed (and are not a function of the treatment). ${ }^{1}$ This amounts to assuming a sequential conditional independence (or ignorability) assumption w.r.t. the mediator and the treatment. It requires (i) that the potential outcomes and the treatment are independent given the observed covariates, and (ii) that the potential outcomes and the mediator are indepen-

() 2016 American Statistical Association Journal of Business \& Economic Statistics January 2016, Vol. 34, No. 1 DOI: 10.1080/07350015.2015.1017644 Color versions of one or more of the figures in the article can be found online at www.tandfonline.com/r/jbes.

${ }^{1}$ If some confounders of the mediator are a function of the treatment, identification becomes more difficult; see for instance the discussion in Robins (2003), Avin, Shpitser, and Pearl (2005), Albert and Nelson (2011), Imai and Yamamoto (2013), and Huber (2014). 
dent given the covariates and the treatment; see for instance Imai, Keele, and Yamamoto (2010b) or the closely related assumptions in Pearl (2001) and Flores and Flores-Lagunes (2009). Under this condition, various estimators of causal mechanisms have been proposed that differ in terms of imposed functional form assumptions. One popular approach is to estimate direct and indirect effects by a system of linear equations characterizing the mediator (as function of the treatment and the covariates) and the outcome (as function of the mediator, treatment, and the covariates). Consistency of the estimator requires that both equations are correctly specified and thus linear, which appears ill-suited, for example, for binary mediators/outcomes. In contrast, g-computation (suggested by Robins 1986, and considered in the context of direct and indirect effects, for instance, in Zheng and van der Laan 2012) also allows for nonlinear mediator and outcome models. It estimates the parameters of interest by the sample analogs of the so-called mediation formula; see for instance Pearl (2001). The latter expresses direct and indirect effects as functions of the conditional mean outcomes (given the mediator, the treatment, and the covariates) and the conditional mediator densities (given the treatment and the covariates), which are estimated by maximum likelihood methods. Again, if either the conditional means or the conditional densities are incorrectly specified, g-computation is in general inconsistent.

Several more flexible semiparametric methods have been suggested more recently. Huber (2014) used inverse probability weighting (henceforth IPW; see the seminal paper by Horvitz and Thompson (1952)) by treatment propensity scores (given the mediator and the covariates or the covariates only, respectively) to estimate causal mechanisms. Furthermore, "multiply robust" estimators (the equivalent to doubly robust estimation in standard treatment effect models) have been proposed by Tchetgen Tchetgen and Shpitser (2012) (based on the efficient influence function) and Zheng and van der Laan (2012) (based on a targeted maximum likelihood approach). Although results about the asymptotic behavior of most estimators are available, little is known about their comparative performance in finite samples and their robustness to misspecification (which is almost unavoidable in empirical applications) of model components.

The contribution of this article is to thoroughly assess the finite sample behavior of a range of common estimators of direct and indirect effects under the assumption of sequential conditional independence. ${ }^{2}$ To this end, we apply a sophisticated simulation design that is based on a large empirical dataset, an approach also advocated in Huber, Lechner, and Wunsch (2013) and named "Empirical Monte Carlo study" (EMCS) therein. To be concise, we use linked jobseeker-caseworker data from Switzerland, which allow disentangling the effect of treatment "counseling style of caseworkers" (rigorous vs. cooperative style) on jobseekers' employment into a direct effect and an indirect effect running via assignment to active labor market

\footnotetext{
${ }^{2}$ While the finite sample performance of estimators of direct and indirect effects appears underresearched up to date, several simulation studies compare various parametric and semiparametric estimators of total (average) effects; see, for instance, Frölich (2004), Lunceford and Davidian (2004), Zhao (2004), Huber, Lechner, and Wunsch (2013), and Busso, DiNardo, and McCrary (in press a,b). The latter two papers base the simulations (at least partly) on real-world data.
}

policies (see the companion paper Huber, Lechner, and Mellace, 2014, for an empirical evaluation). Basing the simulations on empirical data is hopefully more closely related to real-world applications than conventional Monte Carlo designs based on completely artificial (and potentially arbitrary) data-generating processes (DGPs). ${ }^{3}$ We vary several simulation parameters such as sample size, effect heterogeneity, selection into the mediator, the share of individuals assigned to active labor market policies (the mediator), and the type of outcome (binary and nonbinary), which entails a large variety of models considered. The estimators investigated include IPW (Huber 2014), "multiply robust" estimation (influence function-based estimation of Tchetgen Tchetgen and Shpitser, 2012, and targeted maximum likelihood estimation (MLE) of Zheng and van der Laan 2012), estimation by a system of linear equations (see Baron and Kenny 1986), g-computation, parametric estimation using the "mediation" package for R by Tingley et al. (2014) (which is consistent under the same assumptions as g-computation), and a further flexible parametric approach based on regressions in the subpopulations defined by the treatment state.

Our results do not point to a uniformly best performing estimator in all simulations in terms of the root mean squared error (RMSE). Often (but not always), the differences between particular estimators are minor and their relative performance varies with the features of the DGP. For instance, g-computation most frequently dominates the other methods (followed by targeted MLE) when estimating direct effects, but several estimators perform similarly well. With regard to the indirect effects, flexible parametric regression always dominates in the nonbinary outcome case, but only under effect homogeneity when the outcome is binary, while IPW and influence function-based estimation are preferable under effect heterogeneity and binary outcomes. We also investigate the performance of each method when jointly estimating direct and indirect effects, based on the norm of the joint RMSE matrix. For the nonbinary outcome, g-computation and flexible parametric estimation dominate; for the binary outcome, g-computation and influence-based estimation are slightly better than IPW and targeted MLE. Overall, g-computation is the preferred method in our simulations.

The remainder of the article is organized as follows. The next section describes the parameters of interest and the sequential conditional independence (or ignorability) assumption required

${ }^{3}$ Recently such a Monte Carlo approach has been criticized by Advani and Sloczyński (2013), who compared the performance of various estimators in the so-called LaLonde (1986) data with their performance in different Monte Carlo designs. However, their paper suffers from several drawbacks: (a) The experimental estimate in the LaLonde (1986) data is taken as the true effect. In reality, however, it is only an unbiased estimate of the true effect. The ordering of the estimators is therefore disturbed if some estimators are closer to the true value than the experimental estimate (which is random). (b) The LaLonde data are rather small. This implies (i) a noisy estimate of the estimated treatment model used for the placebo simulations, and (ii) that the "population" to draw from is rather small and generated samples may not reflect the statistical concept of a "sample drawn from a infinite population" on which inference measures are based upon. (c) The conditional independence of the treatment is most likely not valid in the LaLonde data, given the limited number of observed covariates. However, it is by construction valid in the simulated data of Advan and Sloczyński (2013). Thus, the idea on which the EMCS is based, namely that the selection and outcome processes in the true world and the EMCS data are very similar, is likely violated for the selection process into treatment. 


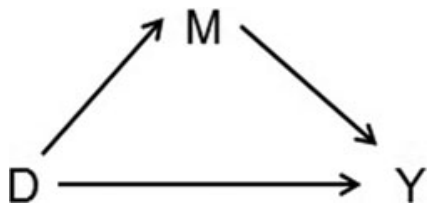

Figure 1. Graphical illustration of the mediation framework.

for the consistency of the estimators. Section 3 discusses the various classes of estimators considered in the simulations. Section 4 describes the empirical Monte Carlo design and the data it is based upon. The main results are presented in Section 4, while the further results and descriptive statistics are provided in the Appendix. Section 5 concludes.

\section{PARAMETERS OF INTEREST AND IDENTIFYING ASSUMPTIONS}

Denote by $D$ a binary intervention or treatment and by $Y$ the outcome variable of interest. Mediation analysis aims at disentangling the causal effect of $D$ on $Y$ into a direct component and an indirect effect operating through some mediator $M$, which may be discrete or continuous. Figure 1 provides a graphical illustration of the evaluation framework considered (where arrows represent causal effects from one variable to another), however, omitting any confounders. To define the parameters of interest, we use the potential outcome framework advocated by Rubin (1974) (among many others) and considered in the direct and indirect effects framework for instance by Rubin (2004), Ten Have et al. (2007), and Albert (2008). Let $M(d), Y(d, M(d))$ denote the potential mediator state and the potential outcome under treatment $d \in\{0,1\}$. For each unit only one of the two potential mediator states and outcomes, respectively, is observed, because the realized outcome and mediator values are $M=D \cdot M(1)+(1-D) \cdot M(0)$ and $Y=D \cdot Y(1, M(1))+(1-D) \cdot Y(0, M(0))$

The (total) average causal effect is given by $\Delta=$ $E[Y(1, M(1))-Y(0, M(0))]$. Exogenously varying the treatment but keeping the mediator fixed at its potential value for some $d \in\{0,1\}$ gives the (average) direct effect:

$$
\theta(d)=E[Y(1, M(d))-Y(0, M(d))], \quad d \in\{0,1\} .
$$

The (average) indirect effects is obtained by exogenously shifting the mediator to its potential values under treatment and nontreatment but keeping the treatment fixed at $d:^{4}$

$$
\delta(d)=E[Y(d, M(1))-Y(d, M(0))], \quad d \in\{0,1\} .
$$

\footnotetext{
${ }^{4}$ Pearl (2001) referred to these parameters as natural direct and indirect effects, Robins and Greenland (1992) and Robins (2003) as total or pure direct and indirect effects, and Flores and Flores-Lagunes (2009) as net and mechanism average treatment effects, respectively.
}

Note that the average causal effect is the sum of the direct and indirect effects defined upon opposite treatment states

$$
\begin{aligned}
\Delta= & E[Y(1, M(1))-Y(0, M(0))] \\
= & E[Y(1, M(0))-Y(0, M(0))]+E[Y(1, M(1)) \\
& -Y(1, M(0))]=\theta(0)+\delta(1) \\
= & E[Y(1, M(1))-Y(0, M(1))]+E[Y(0, M(1)) \\
& -Y(0, M(0))]=\theta(1)+\delta(0) .
\end{aligned}
$$

This follows from adding and subtracting $E[Y(0, M(1))]$ after the second and $E[Y(1, M(0))]$ after the fourth equalities. Using the notation $\theta(1), \theta(0)$ and $\delta(1), \delta(0)$ points to potential effect heterogeneity w.r.t. the treatment, that is, interaction effects between the treatment and the mediator. However, any effect cannot be identified without further assumptions, because either $Y(1, M(1))$ or $Y(0, M(0))$ is observed for any individual, whereas $Y(1, M(0))$ and $Y(0, M(1))$ are never observed.

A good part of the literature based identification of direct and indirect effects on a sequential conditional independence (or ignorability) assumption of the treatment and the mediator; see for instance Imai, Keele, and Yamamoto (2010b), Tchetgen Tchetgen and Shpitser (2012), and Huber (2014). To this end, let $X$ denote a vector of observed covariates that potentially confound the treatment and/or mediator effect(s) on the outcome.

Assumption 1 (conditional independence of the treatment). $\left\{Y\left(d^{\prime}, m\right), M(d)\right\} \perp D \mid X=x$ for all $d^{\prime}, d \in\{0,1\}$ and $m, x$ in the support of $M, X$.

Assumption 1 states that conditional on $X$, the treatment is independent of the potential mediator states and the potential outcomes, implying that there are no unobserved confounders jointly affecting the treatment on the one hand and the mediator and/or the outcome on the other hand given $X$. This is referred to as conditional independence, selection on observables, or exogeneity in the treatment evaluation literature (see, for instance, Imbens 2004).

Assumption 2 (conditional independence of the mediator). $Y\left(d^{\prime}, m\right) \perp M \mid D=d, X=x$ for all $d^{\prime}, d \in\{0,1\}$ and $m, x$ in the support of $M, X$.

Assumption 2 states that conditional on $D$ and $X$, the mediator is independent of the potential outcomes. This implies that there exist no unobserved confounders jointly causing the mediator and the outcome given the treatment and the covariates. Assumption 2 would for instance be violated if unobserved pretreatment variables affected both $M$ and $Y$ directly, that is, not only through $D$ and $X$.

Assumption 3 (common support). $\operatorname{Pr}(D=d \mid M=m, X=$ $x)>0$ for all $d \in\{0,1\}$ and $m, x$ in the support of $M, X$.

Assumption 3 is a common support restriction. The conditional probability to be treated given $M, X$, henceforth referred to as propensity score, ${ }^{5}$ must be larger than zero in either treatment state. This implies that $\operatorname{Pr}(D=d \mid X=x)>0$

\footnotetext{
${ }^{5}$ Rosenbaum and Rubin (1983) defined the propensity score as the probability of being treated conditional on pretreatment variables. Therefore, defining $\operatorname{Pr}(D=1 \mid M, X)$ as "propensity score" is somewhat unconventional since we also condition on posttreatment variables (i.e., the mediators). We thank an anonymous referee for pointing this out.
} 
must hold, too. By Bayes' theorem, Assumption 3 also entails $\operatorname{Pr}(M=m \mid D=d, X=x)>0$ or, in the case of a continuous $M$, that the conditional density of $M$ given $D, X$ is larger than zero: $f_{M \mid D, X}(m, d, x)>0$. In other words, conditional on $X$, the mediator state must not be deterministically affected by the treatment, otherwise comparable units in terms of mediator values across treatment states would not exist.

Under these assumptions, the direct and indirect effects are identified by the so-called mediation formulas, see for instance Pearl (2001) and Imai, Keele, and Yamamoto (2010b), which represent the direct and indirect effects as functions of the conditional mean of $Y$ given $D, M, X$ and the conditional density of $M$ given $D, X$ :

$$
\begin{aligned}
& \theta(d)= E_{X}\left[E_{M \mid D=d, X=x}[E[Y \mid D=1, M=m, X=x]\right. \\
&-E[Y \mid D=0, M=m, X=x] \mid D=d, X=x]], \\
& \delta(d)=E_{X}\left[E_{M \mid D=1, X=x}[E[Y \mid D=d, M=m, X=x]\right. \\
&\mid D=1, X=x]] \\
&-E_{X}\left[E_{M \mid D=0, X=x}[E[Y \mid D=d, M=m, X=x]\right. \\
&\mid D=0, X=x]] .
\end{aligned}
$$

Huber (2014) showed that the parameters of interest may alternatively be expressed as functions of the treatment propensity scores $\operatorname{Pr}(D=1 \mid M, X)$ and $\operatorname{Pr}(D=d \mid X)$, which is useful for estimation based on IPW.

$$
\begin{aligned}
\theta(d)= & E\left[\left(\frac{Y D}{\operatorname{Pr}(D=1 \mid M, X)}-\frac{Y(1-D)}{1-\operatorname{Pr}(D=1 \mid M, X)}\right)\right. \\
& \left.\times \frac{\operatorname{Pr}(D=d \mid M, X)}{\operatorname{Pr}(D=d \mid X)}\right], \\
\delta(d)=E & {\left[\frac { Y I \{ D = d \} } { \operatorname { P r } ( D = d | M , X ) } \left(\frac{\operatorname{Pr}(D=1 \mid M, X)}{\operatorname{Pr}(D=1 \mid X)}\right.\right.} \\
& \left.\left.-\frac{1-\operatorname{Pr}(D=1 \mid M, X)}{1-\operatorname{Pr}(D=1 \mid X)}\right)\right] .
\end{aligned}
$$

Note that the simulations focus on the estimation of $\theta(0)$ and $\delta(1)$, while $\theta(1)$ and $\delta(0)$ are not considered. As the estimation problems are symmetric, we would, however, expect that the overall performance in estimating either $\theta(0), \delta(1)$ or $\theta(1), \delta(0)$ is similar within a particular econometric method.

Albeit not required for nonparametric identification, a subset of parametric estimators considered below is only consistent if average treatment-mediator interaction effects on the outcome are ruled out. This implies that average direct effects are homogeneous in the value of the mediator, as formally stated in Assumption 4:

Assumption 4. $E[Y(1, m) \mid X=x]-E[Y(0, m) \mid X=x]=$ $E\left[Y\left(1, m^{\prime}\right) \mid X=x\right]-E\left[Y\left(0, m^{\prime}\right) \mid X=x\right]$ for all $m, m^{\prime}, x$ in the support of $M, X$.

It follows that $\theta(1)=\theta(0)=\theta$, because the direct effects are identical under $M(1)$ and $M(0)$, and $\delta(1)=\delta(0)=\delta$, because $\delta=\Delta-\theta$. We refer to Assumption 4 as "no interaction" in the spirit of Robins (2003), who assumed no interaction effects (between D and M) at the individual level, whereas we impose this restriction at the population level conditional on $\mathrm{X}$.

\section{ESTIMATORS}

\subsection{Overview}

This section introduces the parametric and semiparametric estimators of direct effects investigated in the simulations. The parametric methods include estimation by a system of linear equations (see Baron and Kenny 1986), a more flexible parametric approach based on regressions within each treatment state, g-computation (suggested by Robins (1986), considered in the context of direct/indirect effects for instance by Zheng and van der Laan (2012)), and estimation based on simulating potential mediators and outcomes using the "mediation" package for R by Tingley et al. (2014). Concerning semiparametric estimation, we consider IPW (Huber 2014), and "multiply robust" estimation based on the efficient influence function (Tchetgen Tchetgen and Shpitser (2012); and targeted maximum likelihood, Zheng and van der Laan (2012)) for assessing direct and indirect effects.

\subsection{Parametric Methods}

3.2.1 OLS Estimation of a System of Linear Equations. Following Baron and Kenny (1986), most of the earlier studies on mediation have estimated direct and indirect effects based on linear equations characterizing the outcome and the mediator. When allowing for observed confounders, this amounts to assuming the following models for the conditional expectations of the outcome and the mediator:

$$
\begin{aligned}
E[Y \mid D, M, X] & =\alpha_{0}+\alpha_{D} D+\alpha_{M} M+X^{\prime} \alpha_{X}, \\
E[M \mid D, X] & =\beta_{0}+\beta_{D} D+X^{\prime} \beta_{X},
\end{aligned}
$$

where $\alpha_{0}, \alpha_{D}, \alpha_{M}, \alpha_{X}$ denote the constant and the coefficients on $D, M, X$ in the outcome equation and $\beta_{0}, \beta_{D}, \beta_{X}$ the constant and the coefficients on $D, X$ in the mediator equation. By its parametric assumptions, this simple model does not allow for interactions of $D$ and $M$ or $X, D$, and $M$ and therefore implies Assumption 4 (along with homogeneous $\theta, \delta$ ).

Throughout the discussion, we assume iid random sampling from a large population and that all moments required for $M$ and $Y$ exist and can be consistently estimated by sample analogs. Let $\hat{\alpha}_{D}, \hat{\alpha}_{M}, \hat{\beta}_{D}$ denote the OLS estimates of $\alpha_{D}, \alpha_{M}$, and $\beta_{D}$, respectively. Then, the estimated direct effect, $\hat{\theta}$, corresponds to $\hat{\alpha}_{D}$ (the partial effect of $D$ on $Y$ after controlling for $M, X$ ) while the indirect effect $\hat{\delta}$ corresponds to $\hat{\beta}_{D} \cdot \hat{\alpha}_{M}$, that is, the first-stage effect of $D$ on $M$ times the second-stage effect of $M$ on $Y$. The drawback of this approach is its general inconsistency under nonlinear mediator or outcome models and/or in the presence of interaction effects. Concerning the latter issue, such models could, however, be easily made more flexible by including interaction terms between $M$ and $D$; see the discussion in Imai, Keele, and Yamamoto (2010b) and Imai, Keele, and Tingley (2010a).

3.2.2 An Alternative, More Flexible Parametric Estimator. As an alternative to using a system of linear equations, we also consider a parametric approach that is somewhat more flexible in terms of functional form assumptions because it estimates separate outcome models across treatment states. Specifically, the conditional mean outcomes $E[Y \mid D=1, M=m, X=x]$ and 
$E[Y \mid D=0, M=m, X=x]$ are estimated by separate parametric regressions in the subpopulations with $D=1$ and $D=0$, with $\hat{\mu}_{Y}(1, m, x), \hat{\mu}_{Y}(0, m, x)$ denoting the respective estimates. The estimated direct effect is obtained by their sample average difference:

$$
\hat{\theta}=\frac{1}{n} \sum_{i=1}^{n}\left\{\hat{\mu}_{Y}\left(1, M_{i}, X_{i}\right)-\hat{\mu}_{Y}\left(0, M_{i}, X_{i}\right)\right\}
$$

where $i$ denotes the index of an observation in an iid sample of size $n(i \in\{1, \ldots, n\})$.

In contrast to the linear model of Section 3.2.1, this approach flexibly allows for interactions between $D$ and $X$. However, consistency requires the satisfaction of Assumption 4, so that heterogeneity in average direct effects across mediator values given $X$ is ruled out. To see how assuming a constant average direct effect across different $m$ conditional on $X$ permits consistent estimation of $\theta$, note that the population analog of (10) is

$$
\begin{aligned}
& E_{X, M}\{E[Y \mid D=1, M=m, X=x] \\
&-E[Y \mid D=0, M=m, X=x]\} \\
&= E_{X}\left\{E_{M \mid X=x}[E[Y(1, m) \mid D=1, M=m, X=x]\right. \\
&-E[Y(0, m) \mid D=0, M=m, X=x]]\} \\
&= E_{X}\left\{E_{M \mid X=x}[E[Y(1, m) \mid D=1, X=x]\right. \\
&-E[Y(0, m) \mid D=0, X=x]]\} \\
&= E_{X}\left\{E_{M \mid X=x}[E[Y(1, m) \mid X=x]-E[Y(0, m) \mid X=x]]\right\} \\
&= E_{X}\{E[Y(1, M) \mid X=x]-E[Y(0, M) \mid X=x]\}=\theta,(11)
\end{aligned}
$$

where the first equality follows from the law of iterated expectations and the fact that $E[Y \mid D=d, M=m, X=x]=$ $E[Y(d, m) \mid D=d, M=m, X=x]$, the second from Assumption 2, the third from Assumption 1, the fourth from the law of iterated expectations, and the fifth from Assumption 4 and the law of iterated expectations.

Concerning the indirect effect, one may first estimate the total causal effect using estimates of $E[Y \mid D=1, X=x]$ and $E[Y \mid D=0, X=x]$, which are denoted by $\hat{\phi}_{Y}(1, x), \hat{\phi}_{Y}(0, x)$. The latter come from separate parametric regressions in the subpopulations with $D=1$ and $D=0$. The indirect effect is then estimated as the difference between the total and the direct effect:

$$
\hat{\delta}=\frac{1}{n} \sum_{i=1}^{n}\left\{\hat{\phi}_{Y}\left(1, X_{i}\right)-\hat{\phi}_{Y}\left(0, X_{i}\right)-\hat{\theta}\right\} .
$$

In the simulations, we adapt the outcome specification to the distribution of $Y$, using linear and probit models for the nonbinary and binary outcomes, respectively. Consistency relies on the correct specification of the models and the absence of treatment-mediator interactions.

3.2.3 g-Computation. In contrast to the previous two approaches, g-computation (going back to Robins 1986) allows for arbitrary treatment-mediator interactions and estimates the mediation formulas in (4) and (5) based on MLE. Let $\hat{\mu}_{Y}(d, m, x)$, $\hat{f}(m \mid d, x)$ denote the estimates of the conditional mean outcome $E[Y \mid D=1, M=m, X=x]$ and the conditional mediator density $f_{M \mid D=d, X=x}(m)$ (or conditional probability $\operatorname{Pr}(M=m \mid D=$ $d, X=x)$ if the mediator is discrete). The g-computation estimators of the direct and indirect effects are given by

$\hat{\theta}(d)=\frac{1}{n} \sum_{i=1}^{n}\left\{\left[\hat{\mu}_{Y}\left(1, M_{i}, X_{i}\right)-\hat{\mu}_{Y}\left(0, M_{i}, X_{i}\right)\right] \hat{f}\left(M_{i} \mid d, X_{i}\right)\right\}$,

$$
\hat{\delta}(d)=\frac{1}{n} \sum_{i=1}^{n}\left\{\hat{\mu}_{Y}\left(d, M_{i}, X_{i}\right)\left[\hat{f}\left(M_{i} \mid 1, X_{i}\right)-\hat{f}\left(M_{i} \mid 0, X_{i}\right)\right]\right\} .
$$

(see also Zheng and van der Laan 2012, sec. 4.2, for the direct effect) In general, both the parametric models for $E[Y \mid D=$ $1, M=m, X=x]$ and $f_{M \mid D=d, X=x}(m)$ need to be correctly specified for consistency.

3.2.4 Estimation Based on Simulating Potential Mediators and Outcomes. The estimation approach of Tingley et al. (2014) as implemented in the "mediation" package for R is based on (a) estimation of the mediator and outcome models and (b) simulation of potential mediators and outcomes according to the estimated models to (c) estimate direct and indirect effects. In particular, the idea is to simulate the potential outcomes $Y(d, M(1-d))$, which can never be observed, but are required for defining the direct and indirect effects in (1) and (2). Among others, the "mediation" package offers the following bootstrap algorithm for effect estimation and inference (see the description in Imai, Keele, and Tingley 2010a):

1. Draw a large number of bootstrap samples out of the original data (the default of the package is 1000 bootstrap samples).

2. In each bootstrap sample: (i) fit models for the observed outcomes and mediators; (ii) simulate the potential values of the mediators according to the parameter estimates in (i) and the mediator model; (iii) simulate the potential outcomes given the simulated values of the mediator, the parameter estimates in (i), and the outcome model; and (iv) estimate the direct and indirect effects based on the simulated analogs of (1) and (2).

3. Take averages of the estimates over all bootstrap samples to obtain the estimated direct and indirect effects $\hat{\theta}, \hat{\delta}$. and confidence intervals.

Assuming, for instance, linear mediator and outcome models with additive error terms, let $\hat{\mu}_{Y}(d, m, x)$ and $\hat{\mu}_{M}(d, x)$ denote estimates of $E[Y \mid D=d, M=m, X=x]$, and $E[M \mid D=$ $d, X=x]$, respectively. ${ }^{6}$ Furthermore, denote by $\tilde{Y}_{i, k(b)}(d, m)$, $\tilde{M}_{i, k(b)}(d)$ the $k$ th simulated potential outcome and mediator (mimicking $Y(d, m), M(d)$ ) for observation $i$ in bootstrap sample $b$ under treatment and mediator values $d, m$. The simulated potential mediators are obtained by

$$
\tilde{M}_{i, k(b)}(d)=\hat{\mu}_{M}\left(d, X_{i(b)}\right)+\tilde{\epsilon}_{i, k(b)}, \quad d \in\{1,0\},
$$

where $X_{i(b)}$ denotes the covariate values of individual $i$ in bootstrap sample $b$ and $\tilde{\epsilon}_{i, k(b)}$ is the $k$ th simulated error for this individual, drawn from the error distribution imposed by the parametric mediator model (e.g., standard normal). That is, $k$ indexes

${ }^{6}$ This example is only chosen for illustrative purposes. Note that the estimation approach of Tingley et al. (2014) also allows for nonlinear models with nonadditive error terms. 
a particular simulation replication of the potential mediator conditional on observation $i$ and satisfies $k \in\{1, \ldots, K\}$, with $K$ being the number of replications per observation. $\tilde{M}_{i, k(b)}(d)$ is plugged into the outcome model and again, a simulated error term $\tilde{u}_{i, k(b)}$ is added to simulate the potential outcomes:

$$
\begin{array}{r}
\tilde{Y}_{i, k(b)}\left(d^{\prime}, M(d)\right)=\hat{\mu}_{Y}\left(d^{\prime}, \tilde{M}_{i, k(b)}(d), X_{i(b)}\right)+\tilde{u}_{i, k(b)}, \\
d, d^{\prime} \in\{1,0\} .
\end{array}
$$

The direct and indirect effects in bootstrap sample $b$, denoted by $\hat{\theta}_{b}(d)$ and $\hat{\delta}_{b}(d)$, are obtained by averaging over simulations and observations:

$$
\begin{aligned}
& \hat{\theta}_{b}(d)=\frac{1}{n K} \sum_{i(b)=1}^{n} \sum_{k(b)=1}^{K}\left\{\tilde{Y}_{i, k(b)}(1, M(d))-\tilde{Y}_{i, k(b)}(0, M(d))\right\} \\
& \hat{\delta}_{b}(d)=\frac{1}{n K} \sum_{i(b)=1}^{n} \sum_{k(b)=1}^{K}\left\{\tilde{Y}_{i, k(b)}(d, M(1))-\tilde{Y}_{i, k(b)}(d, M(0))\right\} .
\end{aligned}
$$

Finally, the estimates of the direct and indirect effects are computed by averaging over the bootstrap samples, with $B$ being the number of bootstraps:

$$
\hat{\theta}(d)=\frac{1}{B} \sum_{b=1}^{B} \hat{\theta}_{b}(d), \quad \hat{\delta}(d)=\frac{1}{B} \sum_{b=1}^{B} \hat{\delta}_{b}(d) .
$$

In our simulations, we consider parametric models for the mediators and outcomes (in the same manner as for g-computation introduced in Section 3.2.3). ${ }^{7}$ Consistency relies on the correct specification of the mediator and outcome models, but does not require Assumption 4 such that treatment-mediator interactions are allowed for.

\subsection{Semiparametric Methods}

3.3.1 Inverse Probability Weighting. Huber (2014) suggested estimation based on IPW, ${ }^{8}$ using normalized versions of the sample analogs of expressions (6) and (7), such that the weights of the observations in either treatment state add up to unity. For example, the estimators of the direct effects under nontreatment and the indirect effect under treatment are given by

$$
\begin{aligned}
\hat{\theta}(0)= & \frac{\sum_{i=1}^{n} Y_{i} D_{i}\left(1-\hat{\rho}\left(M_{i}, X_{i}\right)\right) /\left[\hat{\rho}\left(M_{i}, X_{i}\right)\left(1-\hat{p}\left(X_{i}\right)\right)\right]}{\sum_{i=1}^{n} D_{i}\left(1-\hat{\rho}\left(M_{i}, X_{i}\right)\right) /\left[\hat{\rho}\left(M_{i}, X_{i}\right)\left(1-\hat{p}\left(X_{i}\right)\right)\right]} \\
& -\frac{\sum_{i=1}^{n} Y_{i}\left(1-D_{i}\right) /\left(1-\hat{p}\left(X_{i}\right)\right)}{\sum_{i=1}^{n}\left(1-D_{i}\right) /\left(1-\hat{p}\left(X_{i}\right)\right)}
\end{aligned}
$$

\footnotetext{
${ }^{7}$ We point out that the method of Tingley et al. (2014) even allows for more flexible specifications, namely generalized additive models.

${ }^{8}$ The idea of IPW goes back to Horvitz and Thompson (1952), who first proposed an estimator of the population mean in the presence of nonrandomly missing data.
}

$$
\begin{aligned}
\hat{\delta}(1)= & \frac{\sum_{i=1}^{n} Y_{i} D_{i} / \hat{p}\left(X_{i}\right)}{\sum_{i=1}^{n} D_{i} / \hat{p}\left(X_{i}\right)} \\
& -\frac{\sum_{i=1}^{n} Y_{i} D_{i}\left(1-\hat{\rho}\left(M_{i}, X_{i}\right)\right) /\left[\hat{\rho}\left(M_{i}, X_{i}\right)\left(1-\hat{p}\left(X_{i}\right)\right]\right.}{\sum_{i=1}^{n} D_{i}\left(1-\hat{\rho}\left(M_{i}, X_{i}\right)\right) /\left[\hat{\rho}\left(M_{i}, X_{i}\right)\left(1-\hat{p}\left(X_{i}\right)\right]\right.},
\end{aligned}
$$

where $\hat{\rho}\left(M_{i}, X_{i}\right), \hat{p}\left(X_{i}\right)$ denote the respective estimates of the propensity scores $\operatorname{Pr}\left(D=1 \mid M_{i}, X_{i}\right), \operatorname{Pr}\left(D=1 \mid X_{i}\right)$ based on probit specifications. These semiparametric IPW estimators (into which the propensity scores enter parametrically) can be expressed as sequential GMM estimators where propensity score estimation represents the first step and effect estimation the second step (see Newey 1984). It follows from these results that IPW is $\sqrt{n}$-consistent and asymptotically normal under standard regularity conditions. ${ }^{9}$

3.3.2 Multiply Robust Estimation Based on the Efficient Influence Function. Tchetgen Tchetgen and Shpitser (2012) suggested to base estimation on the sample analogue of the efficient influence or score function (which is zero in expectation) of the effect of interest. To this end, let $\hat{\mu}_{Y}(d, m, x), \hat{f}(m \mid d, x), \hat{p}(x), \hat{\theta}(d, x)$ denote estimates of the conditional mean outcome $E[Y \mid D=d, M=m, X=x]$, the conditional mediator density $f_{M \mid D=d, X=x}(m)$ (or conditional probability if the mediator is discrete), the treatment propensity score $\operatorname{Pr}(D=1 \mid X=x)$, and the conditional direct effect (given $X) \quad \theta(d, x)=E_{M \mid D=d, X=x}[E[Y \mid D=$ $1, M=m, X=x]-E[Y \mid D=0, M=m, X=x] \mid D=d$,

$X=x]$, which may for instance be obtained by regressing $\hat{\mu}_{Y}(1, M, X)-\hat{\mu}_{Y}(0, M, X)$ on $X$ among those with $D=d$. The direct effect under nontreatment is then for instance estimated as

$$
\begin{aligned}
\hat{\theta}(0)= & \frac{1}{n} \sum_{i=1}^{n}\left\{\left[\frac{D_{i} \hat{f}\left(M_{i} \mid 0, X_{i}\right)}{\hat{p}\left(X_{i}\right) \hat{f}\left(M_{i} \mid 1, X_{i}\right)}-\frac{1-D_{i}}{1-\hat{p}\left(X_{i}\right)}\right]\right. \\
& \times\left[Y_{i}-\hat{\mu}_{Y}\left(D_{i}, M_{i}, X_{i}\right)\right]+\frac{1-D_{i}}{1-\hat{p}\left(X_{i}\right)} \\
& \times\left[\hat{\mu}_{Y}\left(1, M_{i}, X_{i}\right)-\hat{\mu}_{Y}\left(0, M_{i}, X_{i}\right)-\hat{\theta}\left(0, X_{i}\right)\right] \\
& \left.+\hat{\theta}\left(0, X_{i}\right)\right\} .
\end{aligned}
$$

As discussed by Zheng and van der Laan (2012), this estimator is (for instance in contrast to g-computation) "multiply robust" in the sense that it remains consistent if only particular subsets of the model specifications are correct. Namely, it needs to hold that at least either (i) $E[Y \mid D, M, X]$ and $\theta(D, X)$, (ii) $E[Y \mid D, M, X]$ and $\operatorname{Pr}(D=1 \mid X)$, or (iii) $\operatorname{Pr}(D=1 \mid X)$ and $f_{M \mid D, X}$ are correctly specified. If all three conditions hold,

${ }^{9}$ However, if the common support Assumption 3 is close to being violated, estimation in finite samples may be unstable due to an explosion of the variance; see for instance Khan and Tamer (2010) and Busso, DiNardo, and McCrary (in press a,b). Furthermore, weighting may be less robust to propensity score misspecification than other classes of estimators, as documented for instance in Kang and Schafer (2007), Waernbaum (2012), and Huber, Lechner, and Wunsch (2013). 
then multiply robust estimation reaches the semiparametric efficiency bound in the limit.

Let $\quad \psi(d, x)=E_{M \mid D=d, X=x}[E[Y \mid D=1, M=m, X=x]$ $\mid D=d, X=x]$ and $\hat{\psi}(d, x)$ denote its estimate. The indirect effect is obtained by

$$
\begin{aligned}
\hat{\delta}(1)= & \frac{1}{n} \sum_{i=1}^{n}\left\{\frac { D _ { i } } { \hat { p } ( X _ { i } ) } \left[Y-\hat{\psi}\left(1, X_{i}\right)-\frac{\hat{f}\left(M_{i} \mid 0, X_{i}\right)}{\hat{f}\left(M_{i} \mid 1, X_{i}\right)}\right.\right. \\
& {\left.\left[Y-\hat{\mu}_{Y}\left(1, M_{i}, X_{i}\right)\right]\right] } \\
& \left.-\frac{1-D_{i}}{1-\hat{p}\left(X_{i}\right)}\left[\hat{\mu}_{Y}\left(1, M_{i}, X_{i}\right)\right]-\hat{\psi}\left(0, X_{i}\right)\right] \\
& \left.+\psi\left(1, X_{i}\right)-\hat{\psi}\left(0, X_{i}\right)\right\} .
\end{aligned}
$$

The estimator is consistent if either (i) $E[Y \mid D, M, X]$ and $\psi(D, X)$, (ii) $E[Y \mid D, M, X]$ and $\operatorname{Pr}(D=1 \mid X)$, or (iii) $\operatorname{Pr}(D=$ $1 \mid X)$ and $f_{M \mid D, X}$ are correctly specified and asymptotically semiparametrically efficient if all three conditions are satisfied.

It is worth mentioning that Tchetgen Tchetgen and Shpitser (2012) (in their sec. 5) offered modifications to their original estimators that aim to improve stability when common support as postulated in Assumption 3 is, at least in finite samples, (close to being) violated. They also presented simulation evidence on the merits of these modifications under a lack of common support due to model misspecification. In our simulations, where serious common support problems do not arise (see the minor differences between estimation with and without trimming influential observations in Section 5), we only consider the original rather than the modified estimators.

3.3.3 Multiply Robust Estimation Based on Targeted Maximum Likelihood. The final method considered is the targeted maximum likelihood estimator (TMLE) ${ }^{10}$ of Zheng and van der Laan (2012), which also possesses the "multiple robustness" property and asymptotically reaches the semiparametric efficiency bound. ${ }^{11}$ For initial estimators of "ingredients" (conditional mean outcomes, mediator densities, treatment propensities) of the likelihood of the parameter of interest (direct or indirect effects), it iteratively maximizes the likelihood along a least favorable submodel through updating the initial estimators. The parameter estimate is obtained by evaluating the parameter map at the optimized estimator of the likelihood. For the direct effect under nontreatment, TMLE relies on updated estimation of $\mu_{Y}(d, m, x)$ and $\theta(0, x)$, using further components of the efficient influence function as predictors for the updates. For ease of exposition, we assume that both the conditional mean outcome $E[Y \mid D, M, X]$ and the conditional direct effect $\theta(0, X)$ are linear functions (even though the procedure can also be applied to nonlinear models). Let $\hat{\mu}_{Y}\left(D_{i}, M_{i}, X_{i}\right)$ be the OLS estimate of the conditional mean outcome for observation $i$. An updated estimate is obtained by

$$
\hat{\mu}_{Y}^{*}\left(D_{i}, M_{i}, X_{i}\right)=\hat{\mu}_{Y}\left(D_{i}, M_{i}, X_{i}\right)+\hat{\gamma}_{1} \hat{B}\left(D_{i}, M_{i}, X_{i}\right),
$$

\footnotetext{
${ }^{10}$ See the seminal paper of van der Laan and Rubin (2006) for further details on TMLE.

${ }^{11}$ We are indebted to Mark van der Laan and Wen Zheng for providing us with their $\mathrm{R}$ code of the estimator and giving helpful advice concerning its implementation.
}

with the predictor $\hat{B}\left(D_{i}, M_{i}, X_{i}\right)=\left[\frac{D_{i} \hat{f}\left(M_{i} \mid 0, X_{i}\right)}{\hat{p}\left(X_{i}\right) \hat{f}\left(M_{i} \mid 1, X_{i}\right)}-\frac{1-D_{i}}{1-\hat{p}\left(X_{i}\right)}\right]$ and $\hat{\gamma}_{1}$ being the coefficient estimate of $\hat{B}(D, M, X)$ when regressing $\hat{\mu}_{Y}(D, M, X)$ on $\hat{B}(D, M, X)$. Second, denote by $\hat{\theta}^{*}(0, X)$ the estimate of the conditional direct effect under nontreatment using $\hat{\mu}_{Y}^{*}(1, M, X)-\hat{\mu}_{Y}^{*}(0, M, X)$ (rather than $\left.\hat{\mu}_{Y}(1, M, X)-\hat{\mu}_{Y}(0, M, X)\right)$, for instance by regressing $\hat{\mu}_{Y}^{*}(1, M, X)-\hat{\mu}_{Y}^{*}(0, M, X)$ on $X$ among the nontreated. An updated estimate of the conditional direct effect is obtained by

$$
\hat{\theta}^{* *}\left(0, X_{i}\right)=\hat{\theta}^{*}\left(0, X_{i}\right)+\hat{\gamma_{2}} \hat{C}\left(D_{i}, X_{i}\right),
$$

with the predictor $\hat{C}\left(D_{i}, X_{i}\right)=\left[\frac{1-D_{i}}{1-\hat{p}\left(X_{i}\right)}\right]$ and $\hat{\gamma}_{2}$ being the coefficient estimate of $\hat{C}(D, X)$ when regressing $\hat{\theta}^{*}(0, X)$ on $\hat{C}(D, X)$. Finally, the TMLE-based direct effect is estimated as

$$
\hat{\theta}(0)=\frac{1}{n} \sum_{i=1}^{n} \hat{\theta}^{* *}\left(0, X_{i}\right) .
$$

Estimation of the indirect effect again uses updated estimation of $\hat{\mu}_{Y}^{*}\left(D_{i}, M_{i}, X_{i}\right)$ as outlined (24), however now using $\hat{B}\left(D_{i}, M_{i}, X_{i}\right)=\left[\frac{D_{i}}{\hat{p}\left(X_{i}\right)}\left(1-\frac{\hat{f}\left(M_{i} \mid 0, X_{i}\right)}{\hat{f}\left(M_{i} \mid 1, X_{i}\right)}\right]\right.$ as predictor. Furthermore, denote by $\hat{\psi}^{*}(D, X)$ the estimate of $\psi(D, X)$ using $\hat{\mu}_{Y}^{*}(1, M, X)\left(\right.$ rather than $\left.\hat{\mu}_{Y}(1, M, X)\right)$. An updated estimate of $\hat{\psi}^{*}\left(D_{i}, X_{i}\right)$ is given by

$$
\hat{\psi}^{* *}\left(D_{i}, X_{i}\right)=\hat{\psi}^{*}\left(D_{i}, X_{i}\right)+\hat{\gamma}_{2} \hat{C}\left(D_{i}, X_{i}\right),
$$

with $\hat{C}\left(D_{i}, X_{i}\right)=\frac{2 D_{i}-1}{\hat{p}\left(X_{i}\right)}$. Finally, the TMLE-based indirect effect is estimated as

$$
\hat{\delta}(1)=\frac{1}{n} \sum_{i=1}^{n}\left\{\hat{\psi}^{* *}\left(1, X_{i}\right)-\hat{\psi}^{* *}\left(0, X_{i}\right)\right\} .
$$

\section{MONTE CARLO DESIGN}

\subsection{Basic Idea of the Simulation Design}

Similar to the approach advocated in Huber, Lechner, and Wunsch (2013), we base our Monte Carlo simulations (at least to a certain extent) on empirical data rather than on completely artificial scenarios in which the DGP is entirely determined by the researcher. In the latter case, the distributions of outcomes, mediators, and covariates as well as selection into the treatment and the mediator often appear quite arbitrary. In our EMCS, observed outcomes, mediators, and covariates (instead of simulated ones) along with the empirically observed selection processes are used with the hope to more closely mimic realworld applications. We nevertheless would like to vary some key parameters in our EMCS approach, such as, for example, the strength of selection into the mediator and the sample size, to consider range of scenarios that are in the best case empirically relevant, albeit the generalizability of our results to other real-world problems cannot be formally guaranteed. Furthermore, the data must be sufficiently large to be able to treat the empirical data as the "population" (which is infinitely large in conventional simulations with artificial DGPs).

We therefore base our EMCS on large-scale Swiss labor market data analyzed by Behncke, Frölich, and Lechner (2010a, 2010b) to estimate total treatment effects. Huber, Lechner, and 
Mellace (2014) used this linked jobseeker-caseworker data for the evaluation of causal mechanisms. Imposing a sequential conditional independence assumption, they disentangle the positive effect of the treatment "counseling style of caseworkers" (rigorous vs. cooperative style) on jobseekers' employment into a direct effect and an indirect effect running via assignment to active labor market policies (e.g., a training program). ${ }^{12}$ Our EMCS is based on a very similar research question with a binary treatment/mediator and consists of three steps: First, we estimate the selection processes into the treatment and the mediator in the data and use them as true propensity scores for the simulations. Second, we repeatedly draw samples of observations with treatment and mediator equal to zero from the actual data and simulate a (pseudo-)treatment and mediator for each draw based on the empirically estimated selection processes. By definition, the true direct and indirect effects are zero (as only observations with zero treatment/mediator are considered) and therefore homogeneous. To also consider heterogeneous effects (with treatment-mediator interactions), we explicitly model the outcome as a function of the treatment, mediator, and covariates (which comes at the cost of imposing more structure in the simulations than in the case of homogeneous zero effects). In addition, we vary further simulation parameters - as for instance the sample size, the strength of selection into the mediator, or the share of mediated observations - to obtain a rich set of different scenarios, all in all 128 DGPs. Third, we apply a range of different estimators to each sample to evaluate their (relative) performance (in terms of RMSE, bias, and variance) across and within the simulation scenarios considered.

In the next subsections we present the details of how the EMCS is implemented. We begin by describing the data sources and the definition of the "population" on which all our simulations are based. We then define the outcomes, treatment, mediator, and the covariates and present descriptive statistics. Finally, we describe the estimation of the "true" model underlying the simulations and the implementation of the various parameters that drive the properties of the DGPs considered.

\subsection{Data and Definition of the "Population"}

The data underlying our EMCS consist of individuals who registered at Swiss regional employment offices anytime during the year 2003, and have been previously considered in Behncke, Frölich, and Lechner (2010b). ${ }^{13}$ Very detailed individual information on the jobseekers is available from the databases of the unemployment insurance system and social security records,

\footnotetext{
${ }^{12}$ The motivation for the analysis in Huber, Lechner, and Mellace (2014) is that the positive employment effect of less cooperative caseworkers found by Behncke, Frölich, and Lechner (2010b) could be due to various causal mechanisms. Rigorous and cooperative caseworkers might for instance differ in the assignment of active labor market programs or the imposition or threat of sanctions, among other dimensions of counseling. Therefore, Huber, Lechner, and Mellace (2014) investigated whether the success of less cooperative case workers is driven by a more effective mix of active labor market programs ("indirect effect") or comes from any remaining counseling dimensions ("direct effect"), possibly including (the threat of) sanctioning and pushing for accepting jobs.

${ }^{13}$ We deleted 102 individuals who registered with the employment office before 2003 but were nevertheless included in the sample of Behncke, Frölich, and Lechner (2010b).
}

including gender, mother tongue, qualification, information on registration and deregistration of unemployment, employment history, participation in active labor market programs, and an employability rating by the caseworker. Regional (labor market relevant) characteristics such as the cantonal unemployment rate were matched to the individual information. Finally, these administrative data were linked to a caseworker survey based on a written questionnaire that was sent to all caseworkers in Switzerland who were employed at an employment office in 2003 and were still active in December 2004 at the time the questionnaire was sent (see Behncke, Frölich, and Lechner 2010b, for further details). The questionnaire included questions about aims, strategies, processes, and organization of the employment office and the caseworkers, among them the treatment variable on caseworker cooperativeness.

The definition of the sample that serves as "population" closely follows the sample selection criteria in Behncke, Frölich, and Lechner (2010b) so that we refer to their paper for further details. The only exception is that we exclude individuals who were registered in the Italian-speaking part of Switzerland to reduce the number of language interaction terms to be included in the model specifications. The final sample therefore consists of 93,076 unemployed persons (rather than 100,222 as in Behncke, Frölich, and Lechner 2010b).

\subsection{Treatment, Mediator, Outcomes, and Covariates}

The caseworker questionnaire contains a question on how important she considers cooperation with the client. As in the main specification of Behncke, Frölich, and Lechner (2010b), we define the treatment $(D)$ to be one if the caseworker reports to pursue a less cooperative strategy and zero otherwise. According to this definition, 47\%/43,669 observations (53\%/49,407 observations) in our population are treated (not treated). ${ }^{14}$ Columns 1-4 in Table 1 report the means of selected observable characteristics across treatment states along with standardized biases (SB) and stars indicating whether two-sample $t$-tests are significant at conventional levels of significance. We see that selection into treatment is rather modest in our subsample of the Behncke, Frölich, and Lechner (2010b) data.

The mediator $(M)$ is defined in terms of the first participation in an active labor market program (ALMP) within nine months after the start of the unemployment spell. Possible ALMP states in the data include job search training, personality course, language skill training, computer training, vocational training, employment program or internship, and nonparticipation in any

\footnotetext{
${ }^{14}$ The exact question was: "How important do you consider the cooperation with the jobseeker, regarding placements in jobs and assignment of active labor market programs?" and could be answered according to a three-point scale (very important, important, less important); see Behncke, Frölich, and Lechner (2010b) for the exact formulation of the response options. It cannot be completely ruled out that due to the self-assessed nature of the treatment, being cooperative or not could mean different things for different personality types of caseworkers, albeit this issue should be mitigated by controlling for the caseworker characteristics included in our analysis (see further below). Furthermore, note that the treatment is defined w.r.t. the cooperativeness of the firs caseworker a jobseeker is assigned to. As mentioned by Huber, Lechner, and Mellace (2014), the same caseworker usually remains in charge of the jobseeker for the entire spell of unemployment.
} 
Table 1. Difference in selected observables between treated and nontreated as well as mediated and nonmediated

\begin{tabular}{|c|c|c|c|c|c|c|}
\hline Characteristics of the unemployed person & \multicolumn{3}{|c|}{ Treatment status } & \multicolumn{3}{|c|}{ Mediator status } \\
\hline Female & 0.43 & 0.45 & 0.04 & 0.48 & 0.44 & $-0.08^{* * *}$ \\
\hline Qualification: unskilled & 0.22 & 0.21 & -0.04 & 0.17 & 0.22 & $0.14^{* * *}$ \\
\hline Qualification: semiskilled & 0.16 & 0.16 & 0.00 & 0.16 & 0.16 & 0.02 \\
\hline Qualification: skilled without degree & 0.04 & 0.04 & -0.01 & 0.04 & 0.04 & 0.02 \\
\hline Employability rating: medium & 0.75 & 0.76 & 0.00 & 0.78 & 0.75 & $-0.05^{* * *}$ \\
\hline Employability rating: high & 0.10 & 0.11 & 0.03 & 0.11 & 0.11 & -0.01 \\
\hline Mother tongue other than German, French, Italian & 0.32 & 0.31 & -0.02 & 0.24 & 0.32 & $0.19^{* * *}$ \\
\hline Number of unemployment spells in last two years & 0.57 & 0.56 & -0.01 & 0.29 & 0.58 & $0.28^{* * *}$ \\
\hline Fraction of time employed in last two years & 0.80 & 0.80 & 0.01 & 0.82 & 0.80 & $-0.08^{* * *}$ \\
\hline Noncooperative caseworker $(D)$ & - & - & - & 043 & 047 & $008^{* * *}$ \\
\hline
\end{tabular}

NOTE: SB stands for standardized bias. We use $*, * *, * * *$ to indicate whether the $p$-value of a two sample $t$-test is smaller than $0.1,0.05$, and 0.01 , respectively.

ALMP. ${ }^{15}$ In the simulations, the mediator is one if the first participation in the nine months window is either a computer course or vocational training (7\%/6601 observations) and zero otherwise (93\%/86,475 observations). $M$ is defined in this way to distinguish between programs targeting relatively high-skilled job seekers versus those for lower-skilled ones. The last three columns in Table 1 provide the means of and test for differences in various observables across mediator states, this time also including the treatment state. Job seekers with $M=1$ have on average significantly higher levels of qualification and employability, are more often female, are less likely to speak one of the official languages in Switzerland as mother tongue, had a lower number of unemployment spells and spent more time in employment in the previous two years, and were less likely assigned to a noncooperative caseworker than job seekers with $M=0$.

We consider two different outcomes $(Y)$ : a binary indicator for employment in month 24 after the start of the unemployment spell and a nonbinary variable consisting of the cumulative months an individual was a jobseeker between (and including) month 10 and month 36 after start. The motivation for using two distinct outcomes is that we would like to assess the performance of the various estimators for both dummy outcomes and outcomes with a richer support. In month 24 about $55 \%$ of the job seekers $(50,725$ observations) were employed. Figure A.1 in Appendix A.1 displays the distribution of the nonbinary outcome variable.

The covariates that we include as controls $(X)$ have also been used in Behncke, Frölich, and Lechner (2010b) and are strong predictors of selection into the treatment and/or the mediator. A difference to Behncke, Frölich, and Lechner (2010b) is, however, that no dummies or interaction terms for the Italianspeaking region are included, because the latter is not used in our simulations. To predict the "true" selection models for the treatment and the mediator (see Section 4.4), we all in all use 34 covariates: the constant, eight jobseeker characteristics (gen-

\footnotetext{
${ }^{15}$ We refer to Gerfin and Lechner (2002) for a more detailed discussion of the features of the various ALMPs in Switzerland.
}

der, qualification, labor market history, and employability-all of which have been found to be relevant confounders in various empirical labor market studies; see, for instance, Lechner and Wunsch 2013), 14 caseworker characteristics possibly related to both counseling style and usage of ALMPs (gender, age, education, experience, organization of jobseeker allocation to caseworkers), the regional unemployment rate, a dummy for French-speaking region, and nine interaction terms with the French-speaking region. Table A.1 in Appendix A.1 provides descriptive statistics for our covariates across treatment and mediator states.

\subsection{Model, Simulation Parameters, and DGPs}

To obtain the "true" models for treatment and mediator selection to be used in the simulations, we estimate probit specifications in which we regress (a) $D$ on $X$ and (b) $M$ on $D$ and $X$ in our "population." The results of (a) and (b) are given in Table A.2 in Appendix A.1. After that, all observations with $D=1$ and $M=1$ (or both) are discarded and henceforth play no role in the simulations, which leaves us with 45,644 observations. The next step is to draw independent Monte Carlo samples with replacement from the "population" of nontreated and nonmediated. We consider sample sizes $(n)$ of 1000 and 4000 observations in our simulations. As all estimators are $\sqrt{n}$ convergent, increasing sample sizes by a factor of four should reduce the standard error by $50 \%$ (in large samples). Thus, our choices of $n$ facilitate checking whether the estimators already attain this asymptotic convergence rate in finite samples.

Having drawn a particular Monte Carlo sample, the next step consists of simulating the (pseudo-)treatment in this sample:

$$
D_{i}=I\left\{X_{i}^{\prime} \hat{\beta}_{\text {pop }}+U_{i}>0\right\},
$$

where $I\{A\}$ is the indicator function, which is one if argument $A$ is satisfied and zero otherwise, $\hat{\beta}_{\text {pop }}$ are the probit coefficient estimates of the treatment model in the "population," and $U_{i}$ is drawn from a standard normal distribution: $U_{i} \sim N(0,1)$. This entails a share of treated, which is on average roughly $45 \%$ in 
Table 2. Pseudo- $R^{2}$ for the DGPs in the nontreated/nonmediated "population"

\begin{tabular}{lcc}
\hline \hline $\begin{array}{l}\text { Selection } \\
\text { into mediator }\end{array}$ & $\begin{array}{c}\text { Share of } \\
M=1\end{array}$ & $\begin{array}{c}\text { Pseudo- } R^{2} \text { of probit } \\
\text { model for } M\end{array}$ \\
\hline Normal & $10 \%$ & 0.010 \\
Normal & $50 \%$ & 0.011 \\
Strong & $10 \%$ & 0.274 \\
Strong & $50 \%$ & 0.296 \\
\hline
\end{tabular}

NOTE: The sample size is 45,644 . The share of $D=1$ is 0.453 and the pseudo- $R^{2}$ of probit model for $D$ is 0.053 in all DGPs.

the simulations. Then, the (pseudo-)mediator is simulated by

$$
M_{i}=I\left\{\lambda\left(D_{i} \hat{\gamma}_{\text {pop }}+X_{i}^{\prime} \hat{\delta}_{\text {pop }}\right)+\alpha+U_{i}>0\right\},
$$

where $\hat{\gamma}_{\text {pop }}$ and $\hat{\delta}_{\text {pop }}$ are the probit coefficient estimates on $D$ and $X$ of the mediator model in the "population' and $V_{i} \sim N(0,1)$. Furthermore and in contrast to the treatment equation, $\lambda$ allows gauging the magnitude of selection into the mediator. In the simulations, we consider $\lambda=1$ (normal selection) and $\lambda=10$ (strong selection). Finally, the additional constant $\alpha$ determines the share of mediated individuals. We set $\alpha$ such that either $10 \%$ or $50 \%$ of the observations have a mediator equal to one. Table 2 gives the pseudo- $R^{2}$ of the mediator model ${ }^{16}$ for the four different DGPs defined by selection into $M(\lambda)$ and the share of mediated $(\alpha)$, and - in the note underneath - the pseudo- $R^{2}$ of the treatment model.

Without further modifications of the outcome, the true direct and indirect effects are homogeneous and equal to zero, as all observations (even the pseudo-treated/mediated ones) are drawn from the population neither treated nor mediated. To also consider heterogeneous effect with treatment-mediator interactions or treatment-mediator-covariate interactions, we also consider simulations in which we explicitly model the outcome as a function of $D, M, X$ (with the caveat that the latter is arbitrary). In the nonbinary outcome case,

$$
\begin{aligned}
\tilde{Y}_{i}= & g\left(Y_{i}, D_{i}, M_{i}, X_{i}\right)=Y_{i}+0.6 D_{i}+0.2 M_{i}+0.4 D_{i} M_{i} \\
& -0.3 D_{i} \mathrm{u} 2 \mathrm{yrs}_{i}^{2}-0.2 M_{i} \mathrm{u}_{2} \mathrm{yrs}_{i}^{2}-0.1 D_{i} M_{i} \mathrm{u} 2 \mathrm{yrs}_{i}^{3},
\end{aligned}
$$

where $\tilde{Y}_{i}$ denotes the newly modeled outcome (while $Y_{i}$ is the outcome initially observed) and $\mathrm{u} 2 \mathrm{yrs}_{i}$ is an element of $X_{i}$, namely the number of unemployment spells in the previous two years (before the start of the unemployment spell in 2003). In the binary outcome case,

$$
\tilde{Y}_{i}=I\left\{g\left(Y_{i}, D_{i}, M_{i}, X_{i}\right)>0\right\} .
$$

The various combinations of the strength of selection into the mediator, the share of mediated observations, binary or nonbinary outcomes, and homogeneous or heterogeneous effects yield all in all 16 different DGPs.

${ }^{16}$ We use the Nagelkerke's (see Nargelkerke 1991) pseudo- $R^{2}$, which is defined as follows:

$$
\text { pseudo- } R^{2}=\frac{1-\left(\frac{L_{\text {intercept }}}{L_{\text {full }}}\right)^{2 / n}}{1-L_{\text {intercept }}^{2 / n}}
$$

where $n$ is the sample size, and $L_{\text {intercept }}$ and $L_{\text {full }}$ are the likelihood functions of the model with the coefficients of all the variables (but the intercept) set to zero and the full model, respectively.
We investigate two further simulation parameters that concern estimation rather than data generation. First, we either use all of the confounders $X$ included in the "true" treatment and mediator models in the various estimators or exclude several variables, namely the dummy for and all interactions with Frenchspeaking region as well as four of the dummies indicating how jobseekers are assigned to caseworkers (by age, employability, region, or other). The latter scenario implies that all estimators are misspecified due to omitted variables. Second, we consider estimation both with and without trimming observations that receive a (too) "large" weight in some estimator. This problem occurs, for instance, in IPW if the propensity scores of some observations are that extreme that they heavily influence the estimate. In particular, a propensity score very close to zero that enters the denominator in IPW may obtain a very large relative weight in finite samples, if further observations with close to zero propensity scores are rare or unavailable, which amounts to a thin support problem. ${ }^{17}$

An econometric issue is that large weights may entail a large variance of the estimator, as results might be quite sensitive to the inclusion or exclusion of influential observations. Several trimming procedures have therefore been proposed in the literature; see, for instance, Busso, DiNardo, and McCrary (in press a) for a survey. Here, we use a trimming rule closely related to that advocated in Huber, Lechner, and Wunsch (2013). Specifically, basing trimming on the IPW weights for the estimation of direct and indirect effects, we require that observations in the minuends and subtrahends of Equations (20) and (21) do not exceed a particular relative weight (denoted by $\omega$ ). Taking for instance the minuend in (21), we discard any observation $i$ that does not satisfy

$$
\frac{D_{i} / \hat{p}\left(X_{i}\right)}{\sum_{i=1}^{n} D_{i} / \hat{p}\left(X_{i}\right)} \leq \omega,
$$

with $\omega \in(0,1]$. In the simulations, we set $\omega=0.05$, so that the relative weight of any observation must not exceed $5 \%$ in any minuend or subtrahend. ${ }^{18}$ In contrast to other trimming methods suggested in the literature, this does not introduce asymptotic bias, as trimming vanishes in large samples if there is asymptotic common support, such that the estimation is asymptotically unbiased. Note that we use this IPW-based trimming rule for all estimators for the sake of comparability and ease of implementation (while a parallel use of different trimming procedures would further complicate the analysis).

Combining the 16 DGPs with estimation using all or omitting some covariates, with/without trimming, and the two different sample sizes yields all in all 128 different scenarios. A final parameter of the EMCS is the number of replications, which is ideally as large as possible to minimize simulation noise. The latter depends negatively on the number of replications and positively on the variance of the estimators. Since the variance is doubled when the sample size is reduced by half, and since

\footnotetext{
${ }^{17}$ Note that the thin support problem vanishes asymptotically if common support holds in the population, as postulated in Assumption 3. See Khan and Tamer (2010) for the implications of asymptotic thin support problems, when common support is close to being violated even in the population.

${ }^{18}$ After discarding observations with too large weights, the remaining weights are normalized again in the IPW estimator to add up to one.
} 
simulation noise is doubled when the number of replications is reduced by half (at least for averages over the iid simulations), we chose to make the number of replications proportional to the sample size. We use 4000 replications for $n=1000$ and 1000 for $n=4000$, as the larger sample size is computationally more expensive and has less variability of the results across different simulation samples than the smaller one.

\section{RESULTS}

\subsection{Overview}

In this section, we first discuss the implementation of the estimators introduced in Section 3 in terms of various modeling options. After that, the results are presented, beginning with simulation features that concern all estimators simultaneously, namely DGP properties (effect homogeneity/heterogeneity, strength of selection, share of mediated observations), estimation with all/omitting some covariates and with/without trimming, and sample size. In a next step, we analyze the impact of different modeling options within particular classes of estimators. Finally, we compare estimators across different classes for an overall assessment. Our conclusions mainly come from analyzing the RMSE of the estimators. Appendix A.2 contains further results concerning the absolute bias and the standard deviation of the estimators, which helps understanding how the RMSEs come about.

\subsection{Implementation of Estimators}

While Section 3 contains the general estimation approaches, we present the details of the particular implementations of the estimators in our EMCS hereafter. In OLS estimation based on a system of linear equations, see Section 3.2.1, all variables enter linearly as displayed in equations (8) and (9) and no modeling options have to be considered. In contrast, for the more flexible parametric estimator with fewer functional form restrictions (Section 3.2.2), g-computation (Section 3.2.3), and estimation based on simulating potential mediators and outcomes (Section 3.2.4), we use different conditional outcome models depending on whether $Y$ is binary or nonbinary. In the latter case, the outcome equations are estimated based on linear models (with Gaussian errors); in the former case, probit specifications are used. For the estimators of Sections 3.2.3 and 3.2.4, the model for the binary mediator is estimated by probit regression of $M$ on $D$ and $X$. Furthermore, we vary the outcome models in terms of treatment-mediator-covariate interactions. That is, we regress $Y$ on either (i) $1, D, M$, and $X$ or (ii) on $1, D, M, X$, and interactions between $D$ and $M, D$ and $X, M$ and $X$, and $D, M$, and $X$. We therefore consider estimation based on both more parsimonious and more flexible outcome models in our simulations when using g-computation or the method relying on simulating potential mediators and outcomes.

Coming to the semiparametric estimators, IPW (Section 3.3.1) is implemented as outlined in Equations (20) and (21), using probit specifications for the treatment propensity scores (without any mediator-covariate interactions). Multiply robust estimation using the efficient influence function (Section 3.3.2) or TMLE (Section 3.3.3) is based on the same mediator and outcome models (with both more parsimonious and more flexible outcome specifications) as g-computation and estimation based on simulating potential mediators and outcomes. In total we therefore consider 11 different estimators of each of the direct and indirect effects and binary/nonbinary outcomes: IPW, two versions of each efficient influence function-based estimation, TMLE, g-computation, and simulation-based estimation, and parametric estimation based on either a system of linear equations or more flexible outcome regression within treatment states.

\subsection{Analysis Within Class of Estimators}

Based on linear regressions, Tables 3 (nonbinary outcome) and 4 (binary outcome) show how different features of the DGPs and model choices affect the RMSEs of estimators within four different classes of methods: IPW, multiply robust, g-computation/simulation-based estimation, and OLS/flexible parametric. ${ }^{19}$ Note that in the binary outcome case, the latter has been multiplied by 100. The upper panels analyze the DGP features that might affect various classes of estimators differentially: effect homogeneity (reference category) versus effect heterogeneity, "normal" (reference category) versus "strong" selection into the treatment, and a smaller $(10 \%$, reference category) versus a larger $(50 \%)$ share of mediated observations. We find that effect heterogeneity by and large increases the RMSE, with the exception of some estimators of the indirect effect under the binary outcome. As Tables A.3-A.6 in Appendix A.2 show, effect heterogeneity entails a larger bias of any estimator, whereas the influence on the estimators' standard deviations is less clear cut in several cases. Second, strong selection always leads to an increase in the RMSE when estimating the indirect effect, while the results are rather ambiguous for the direct effect (going in both directions under the nonbinary outcome and being negative under the binary outcome).$^{20}$ Finally, also the direction of the impact of the share of mediated observations varies over estimators, sample sizes, and types of outcomes.

Next, we consider two features relevant for estimation, namely whether a correct specification or a model with missing variables is used, and whether observations with extreme weights are trimmed. Concerning the latter, the results show that trimming does generally not matter much for the RMSE. This is likely a particularity of our data and the DGPs based

\footnotetext{
${ }^{19}$ The regression-based analysis of various simulation parameters resembles the idea of response surface analyses; see for instance the surveys of Hill and Hunter (1966), Myers, Khuri, and Carter (1989), and Khuri and Mukhopadhyay (2010). Note that if there were no interaction effects across the various simulation parameters, the constant terms in the regressions would correspond to the average RMSEs of the reference estimators in the various classes of estimators (i.e., those methods not represented by any dummy variable), when switching off all other parameter dummies for effect heterogeneity, strong selection, $50 \%$ mediated, misspecification, and trimming.

${ }^{20} \mathrm{~A}$ more thorough investigation revealed that the negative impact of strong selection on the RMSE of direct effect estimation under the binary outcome is almost exclusively driven by the simulations with effect heterogeneity. In this context, it needs to be pointed out that results for the binary outcome under effect heterogeneity appear to be plagued by outliers.
} 
Table 3. OLS analysis of determinants of RMSE for the nonbinary outcome

\begin{tabular}{|c|c|c|c|c|c|c|c|c|}
\hline \multicolumn{9}{|c|}{ Direct effect } \\
\hline & \multicolumn{2}{|c|}{ IPW } & \multicolumn{2}{|c|}{ mult.robust } & \multicolumn{2}{|c|}{ g-comp, simulation } & \multicolumn{2}{|c|}{ OLS, flex. para } \\
\hline Constant & 0.492 & 0.241 & 0.683 & 0.245 & 0.609 & 0.274 & 0.558 & 0.264 \\
\hline Effect heterogeneity & 0.014 & 0.014 & 0.013 & 0.013 & 0.022 & 0.012 & 0.043 & 0.069 \\
\hline Misspecification & -0.001 & 0.013 & -0.091 & 0.010 & -0.053 & 0.006 & 0.001 & 0.020 \\
\hline Trimming & -0.001 & -0.001 & 0.000 & -0.001 & -0.013 & -0.011 & -0.001 & -0.001 \\
\hline TMLE/g-comp. parsimon. & & & -0.147 & -0.001 & -0.096 & -0.024 & & \\
\hline TMLE/g-comp. flexible & & & 0.000 & 0.000 & 0.014 & -0.022 & & \\
\hline DR/simulation parsimon. & & & -0.147 & -0.001 & -0.086 & -0.000 & & \\
\hline OLS flexible & & & & & & & -0.145 & -0.116 \\
\hline Constant & 0.027 & 0.004 & 0.313 & 0.023 & 0.126 & 0.015 & 0.025 & 0.003 \\
\hline Effect heterogeneity & 0.002 & 0.002 & 0.005 & 0.006 & 0.003 & 0.005 & 0.006 & 0.007 \\
\hline Strong selection & 0.059 & 0.034 & 0.207 & 0.041 & 0.089 & 0.034 & 0.049 & 0.034 \\
\hline $50 \%$ mediated & 0.001 & 0.000 & -0.156 & -0.002 & -0.045 & -0.000 & 0.010 & 0.005 \\
\hline Misspecification & -0.002 & 0.004 & -0.098 & 0.010 & -0.025 & 0.008 & -0.003 & 0.003 \\
\hline Trimming & 0.001 & 0.004 & 0.003 & 0.003 & 0.002 & 0.002 & 0.001 & 0.005 \\
\hline TMLE/g-comp. parsimon. & & & -0.209 & -0.012 & -0.086 & -0.013 & & \\
\hline TMLE/g-comp. flexible & & & 0.004 & -0.003 & -0.024 & -0.013 & & \\
\hline DR/simulation parsimon. & & & -0.235 & -0.025 & -0.082 & -0.010 & & \\
\hline OLS flexible & & & & & & & -0.018 & -0.011 \\
\hline Adjusted $R^{2}$ & 0.966 & 0.871 & 0.427 & 0.770 & 0.504 & 0.841 & 0.847 & 0.678 \\
\hline
\end{tabular}

NOTE: "TMLE/g-comp." refers to TMLE in the case of "mult.robust" and to g-computation in the case of "g-comp, simulation." "DR/simulation" refers to doubly robust estimation in the case of "mult.robust" and to simulation-based estimation in the case of "g-comp, simulation."

thereon, which obviously do not entail too influential observations, rather than a general property of the estimators. With regard to misspecification, we expect a variance-bias trade-off, as the (potentially more biased) misspecified models are more parsimonious and may be estimated more precisely. This is by and large confirmed when looking at the respective tables in Appendix A.2: it appears that the two opposing effects cancel each other out to a substantial extent in most cases.

Finally, for several estimators we consider more and less flexible outcome models, which again entail finite sample biasvariance trade-offs. When looking at multiply robust estimation (mult.robust), a clear finding is that using the parsimonious outcome model decreases the RMSE for both influence functionbased estimation and TMLE. Note that influence function-based estimation using the flexible model is the reference category in this comparison. Another striking result is that under the binary outcome, TMLE with a flexible outcome model performs much worse than any other method, which is driven by outliers in some of the simulation draws. Also for g-computation (g-comp) and simulation-based estimation (simulation), the parsimonious outcome model entails a smaller RMSE than the flexible one. When comparing OLS and flexible parametric estimation, however, the opposite holds true, with the latter always outperforming the former, more rigid specification.

\subsection{Comparisons Across Classes of Estimators}

We subsequently compare the best performing estimators of each estimator class (IPW, multiply robust, gcomputation/simulation-based estimation, OLS/flexible parametric) with each other based on their RMSEs, both overall and across different simulation features. For instance, among the estimators based on g-computation/simulation-based estimation, we only include g-computation with the parsimonious outcome model, which always weakly dominates the other estimators in this class; see Tables 3 and 4. For the same reason, we also consider flexible parametric estimation, which clearly outperforms OLS. Among the multiply robust methods, we for the nonbinary outcome use TMLE based on the parsimonious outcome model, which very slightly but consistently dominates (parsimonious) influence function-based estimation (even though the differences in the various scenarios are minor). For the binary outcome, however, we include (parsimonious) influence function-based estimation, which very clearly outperforms TMLE.

Tables 5 and 6 present the results for the four respective estimators of direct and indirect effects. The first column (rel.rmse) provides the relative difference/increase in the RMSE in percent of a particular method when compared to the best performing 
Table 4. OLS analysis of determinants of RMSE for the binary outcome

\begin{tabular}{|c|c|c|c|c|c|c|c|c|}
\hline \multicolumn{9}{|c|}{ Direct effect } \\
\hline Constant & 3.972 & 2.403 & -4.009 & 0.128 & 4.421 & 2.615 & 4.250 & 2.433 \\
\hline Effect heterogeneity & 0.120 & 1.049 & 16.367 & 9.375 & 0.137 & 1.057 & 3.515 & 4.602 \\
\hline Misspecification & -0.065 & 0.057 & 0.672 & -3.337 & -0.073 & -0.072 & 0.037 & -0.027 \\
\hline Trimming & 0.028 & 0.008 & -0.103 & -0.019 & -0.058 & 0.017 & -0.092 & -0.000 \\
\hline TMLE/g-comp. parsimon. & & & -0.209 & -0.008 & -0.429 & -0.092 & & \\
\hline TMLE/g-comp. flexible & & & 32.749 & 16.736 & -0.253 & -0.106 & & \\
\hline DR/simulation parsimon. & & & -0.209 & -0.008 & -0.310 & -0.071 & & \\
\hline OLS flexible & & & & & & & -3.978 & -3.824 \\
\hline \multicolumn{9}{|c|}{ Indirect effect } \\
\hline Constant & 0.210 & 0.052 & 0.585 & -0.109 & 0.471 & 0.025 & -0.027 & -0.350 \\
\hline Effect heterogeneity & -0.190 & -0.067 & 0.240 & 0.252 & -0.143 & 0.015 & 1.398 & 1.029 \\
\hline Strong selection & 0.264 & 0.160 & 0.513 & 0.349 & 0.294 & 0.228 & 0.713 & 0.724 \\
\hline $50 \%$ mediated & 0.045 & 0.042 & -0.004 & 0.116 & 0.031 & 0.071 & 0.150 & 0.419 \\
\hline Misspecification & -0.012 & 0.002 & -0.165 & 0.031 & -0.036 & 0.016 & -0.102 & 0.194 \\
\hline Trimming & 0.019 & 0.006 & 0.051 & 0.007 & 0.023 & -0.002 & 0.079 & 0.002 \\
\hline TMLE/g-comp. parsimon. & & & -0.195 & 0.175 & -0.249 & 0.016 & & \\
\hline TMLE/g-comp. flexible & & & 0.657 & 0.375 & -0.279 & -0.078 & & \\
\hline DR/simulation parsimon. & & & -0.627 & -0.147 & -0.214 & 0.030 & & \\
\hline OLS flexible & & & & & & & -0.196 & -0.367 \\
\hline Adjusted $R^{2}$ & 0.796 & 0.812 & 0.620 & 0.513 & 0.717 & 0.478 & 0.815 & 0.608 \\
\hline
\end{tabular}

NOTE: The binary outcome has been multiplied by 100 . "TMLE/g-comp." refers to TMLE in the case of "mult.robust" and to g-computation in the case of "g-comp, simulation." "DR/simulation" refers to doubly robust estimation in the case of "mult.robust" and to simulation-based estimation in the case of "g-comp, simulation."

Table 5. Relative and absolute RMSEs across simulation features, nonbinary outcome

\begin{tabular}{|c|c|c|c|c|c|c|c|c|c|c|c|c|}
\hline \multirow[b]{2}{*}{ Direct effect } & \multicolumn{2}{|c|}{ All scenarios } & \multicolumn{2}{|c|}{ No trimming } & \multicolumn{2}{|c|}{ homog. effects ${ }^{*}$} & \multicolumn{2}{|c|}{ heterog. effects ${ }^{*}$} & \multicolumn{2}{|c|}{$n=1000^{*}$} & \multicolumn{2}{|c|}{$n=4000^{*}$} \\
\hline & r.rmse & rmse & r.rmse & rmse & r.rmse & rmse & r.rmse & rmse & r.rmse & rmse & r.rmse & rmse \\
\hline TMLE pars. & 1.06 & 0.37 & 1.05 & 0.37 & 0.91 & 0.37 & 1.18 & 0.38 & 1.21 & 0.5 & 0.74 & 0.25 \\
\hline g-comp. pars. & 0 & 0.37 & 0 & 0.37 & 0 & 0.36 & 0 & 0.38 & 0 & 0.49 & 0 & 0.25 \\
\hline flex. parametric & 0.3 & 0.37 & 0.37 & 0.37 & 0.19 & 0.36 & 0.54 & 0.38 & 0.13 & 0.49 & 0.84 & 0.25 \\
\hline IPW & 44.13 & 0.04 & 45.86 & 0.04 & 47.81 & 0.04 & 43.99 & 0.04 & 48.73 & 0.06 & 39.48 & 0.02 \\
\hline influence pars. & 47.41 & 0.04 & 49.22 & 0.04 & 53.2 & 0.04 & 45.42 & 0.04 & 48.64 & 0.06 & 50.51 & 0.03 \\
\hline g-comp. pars. & 36.63 & 0.04 & 38.22 & 0.04 & 34.94 & 0.04 & 41.35 & 0.04 & 32.49 & 0.05 & 50.96 & 0.03 \\
\hline \multirow[t]{2}{*}{ flex. parametric } & 0 & 0.03 & 0 & 0.03 & 0 & 0.03 & 0 & 0.03 & 0 & 0.04 & 0 & 0.02 \\
\hline & \multicolumn{2}{|c|}{ normal sel. ${ }^{*}$} & \multicolumn{2}{|c|}{ strong sel. ${ }^{*}$} & \multicolumn{2}{|c|}{ correct spec. ${ }^{*}$} & \multicolumn{2}{|c|}{ misspec. ${ }^{*}$} & \multicolumn{2}{|c|}{$10 \%$ mediated $^{*}$} & \multicolumn{2}{|c|}{$50 \%$ mediated ${ }^{*}$} \\
\hline flex. parametric & 0.46 & 0.38 & 0.27 & 0.37 & 0.49 & 0.37 & 0.25 & 0.38 & 0.16 & 0.37 & 0.58 & 0.37 \\
\hline Indirect effect & r.rmse & rmse & r.rmse & rmse & r.rmse & rmse & r.rmse & rmse & r.rmse & rmse & r.rmse & rmse \\
\hline IPW & 51.51 & 0.02 & 44.25 & 0.06 & 47.1 & 0.04 & 44.69 & 0.04 & 49.71 & 0.04 & 42.05 & 0.04 \\
\hline influence pars. & 48.64 & 0.02 & 49.39 & 0.06 & 41.8 & 0.04 & 56.17 & 0.04 & 56.6 & 0.04 & 41.95 & 0.04 \\
\hline g-comp. pars. & 38.39 & 0.02 & 38.17 & 0.06 & 28.67 & 0.03 & 47.16 & 0.04 & 44.48 & 0.04 & 32.05 & 0.04 \\
\hline flex. parametric & 0 & 0.01 & 0 & 0.04 & 0 & 0.03 & 0 & 0.03 & 0 & 0.03 & 0 & 0.03 \\
\hline
\end{tabular}

NOTE: "r.rmse," the relative RMSE of any estimator $e$ is defined as $100 *\left(\frac{\text { rmse } e^{-r m s e m i n}}{\text { rmsemin }}\right)$, where rmse $_{e}$ is the RMSE of estimator $e$ and rmse min $_{\text {in }}$ is the minimum RMSE (of the best performing estimator). *Only simulations without trimming are included. 
Table 6. Relative and absolute RMSEs across simulation features, binary outcome

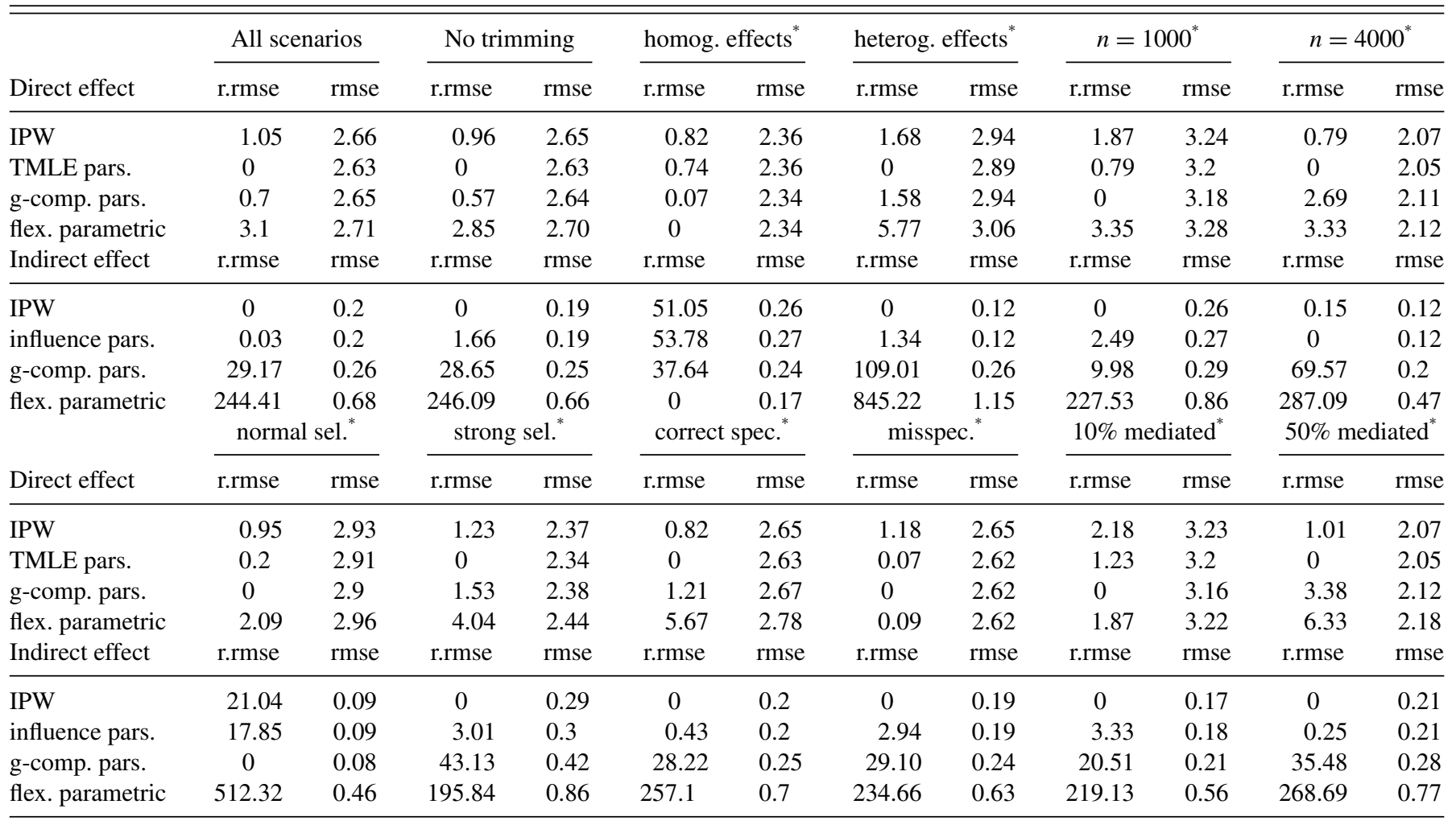

NOTE: "r.rmse," the relative RMSE of any estimator $e$ is defined as $100 *\left(\frac{\text { rmse }_{e}-\text { rmsemin }_{\text {min }}}{\text { rmsemin }_{\text {min }}}\right)$ where rmse $e$ is the RMSE of estimator $e$ and rmse min $_{\text {is }}$ the minimum RMSE (of the best performing estimator). *Only simulations without trimming are included.

estimator with the smallest RMSE (therefore, rel.rmse is 0 for the latter). The second column gives the values of the RMSEs on which this comparison is based. The first panel shows the average RMSEs over all specifications, followed by the results for specifications with and without trimming. As trimming does not importantly affect the RMSEs, all remaining subgroups of specifications (homogeneous/heterogeneous effects, small/large sample size, normal/strong selection, correct/incorrect specification, $10 \% / 90 \%$ mediated) only include simulations without trimming.

The first important observation is that for the direct effects, estimator choice does not matter much. Even though gcomputation dominates most often followed by TMLE (which is best over all scenarios under the binary outcome), all methods have rather similar RMSEs and no estimator has decisively outperformed. For the indirect effects, the picture looks more complex, as the type of outcome variable, that is, binary versus nonbinary, matters crucially. For the nonbinary outcome, the flexible parametric specification of Section 3.2.2 outperforms the competitors in all specifications, at least in terms of the relative RMSE (while absolute differences are often small). All other estimators behave similarly well. For the binary outcome, however, the flexible parametric estimator only dominates under effect homogeneity. In scenarios with heterogeneous effects, the misspecification of the parametric method (which does not allow for effect heterogeneity) seems to be too substantial. Here, IPW and influence function-based estimation (influence pars.) are by and large the best or close to the best estimators, while flexible parametric performs badly and g-computation is somewhere in between. Thus, IPW and the influence function-based method with the parsimonious model turn out to be the recommended estimators in the binary case.

In addition to the RMSEs, we provide the relative and absolute biases and standard deviations of the selected estimators for all scenarios and for simulations without trimming in Table 7. For the direct effects, TMLE has overall the smallest absolute bias. As the relative bias of any other estimator is below $15 \%$ in the scenarios considered, we conclude that all methods perform quite satisfactorily in terms of bias. For the indirect effects, IPW and influence function-based estimation dominate under nonbinary and binary outcomes, respectively. It is noteworthy that under the binary outcome, the absolute bias is comparably large for g-computation, but even much more so for flexible parametric estimation. The latter result is driven by the estimator's poor performance in the simulations with effect heterogeneity, as already noticed for the RMSE. Concerning the standard deviation, all estimators are very similar when considering the direct effects. For the indirect effects, flexible parametric estimation dominates the other methods under the nonbinary outcome but is far worse under the binary outcome. The differences between the other estimators are again moderate.

From an applied perspective, it appears most convenient to have one estimation strategy instead of using two different estimators for the direct and indirect effects. As a measure for the overall performance across effects, Table 8 therefore presents the norms of the joint RMSE matrix of direct and indirect effects for each estimator. Because trimming proved to have little effects, the results are reported for setups without trimming only. For the nonbinary outcome, g-computation and flexible parametric estimation perform similarly well and dominate the remaining 
Table 7. Relative and absolute biases/standard deviations for all scenarios and without trimming

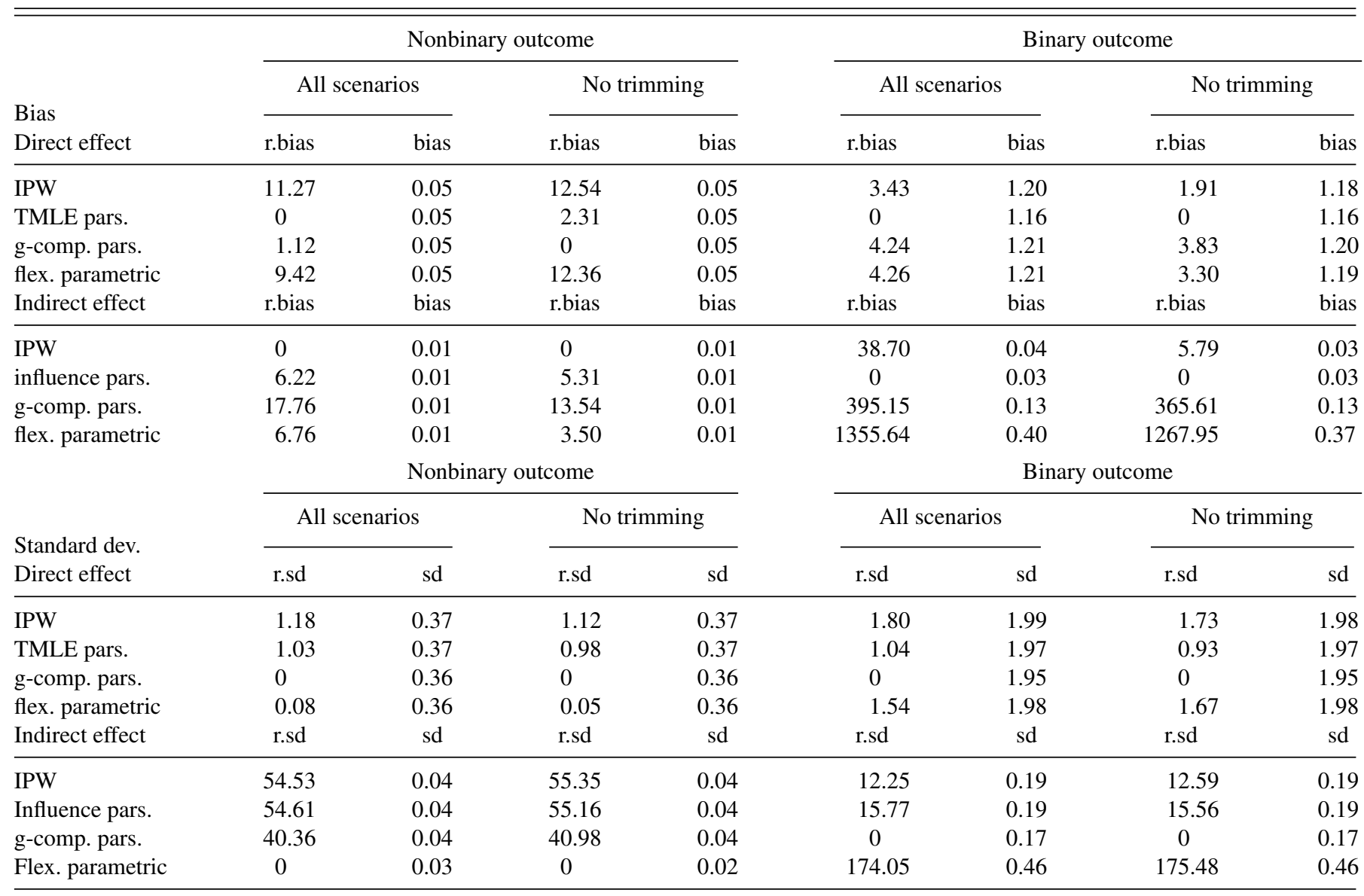

NOTE: "r.bias," the relative absolute bias of any estimator $e$ is defined as $100 *\left(\frac{\text { bias } e^{- \text {biasmin }}}{\text { bias }_{\min }}\right)$, where bias $_{e}$ is the absolute bias of estimator $e$ and bias min $_{\text {in }}$ is the minimum absolute bias (of the best performing estimator). The same applies to "r.sd," the relative standard deviation.

methods in all cases. In the nonbinary case, however, the flexible parametric estimator is only competitive under homogeneous effects and does worse than any other method in the other scenarios (driven by its weakness when estimating indirect effects under effect heterogeneity). Differences between the remaining methods are small, but g-computation and influence-based estimation perform best overall. As g-computation is competitive under both nonbinary and binary outcomes, it appears to be the preferred choice if one would like to use the same method for direct and indirect effects.

Finally, it seems worth considering whether particular combinations of distinct estimators for the direct and indirect effects perform particularly well (in terms of the norm) to assess the relative price to pay for the "convenience" of using just one type of estimator for both effects. Therefore, Table A.7 in Appendix A.2 compares the six respective best combinations of

Table 8. Norms of RMSEs when estimating direct and indirect effects jointly

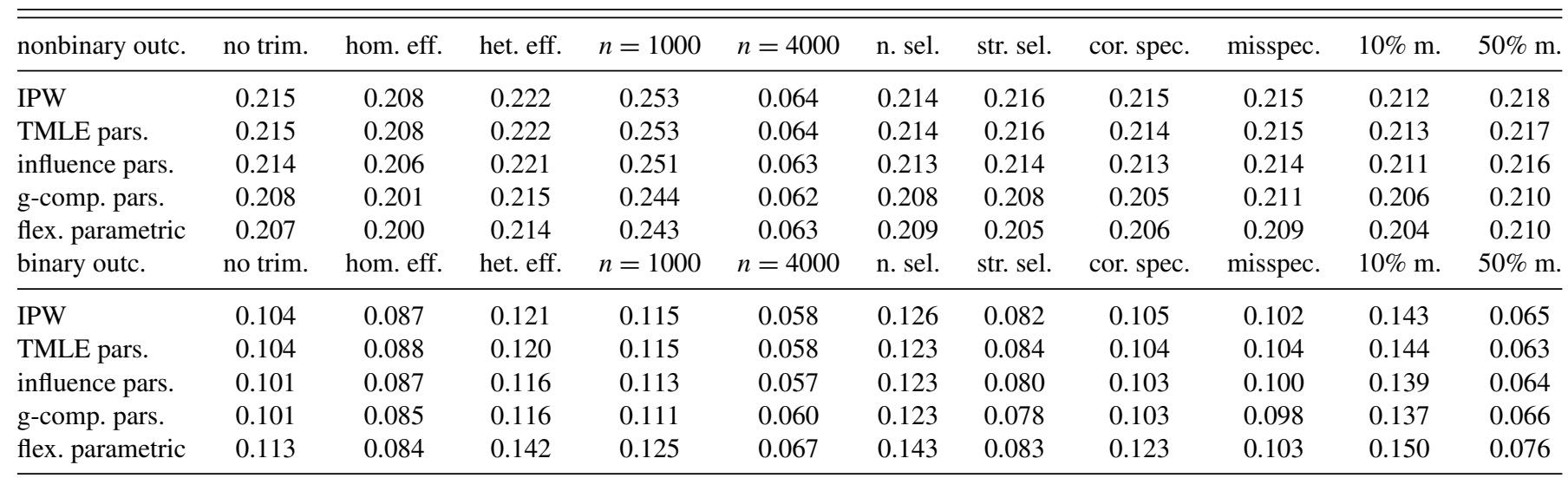

NOTE: Only simulations without trimming are considered. 
distinct estimators to the five pure estimation strategies (using the same method across outcomes). As the differences between the respective best combined and pure methods are very small to nonexistent in any scenario, the price for using the same approach for estimating direct and indirect effects is very low in our EMCS.

\section{CONCLUSION}

This article analyzed the finite sample performance (in terms of the RMSE) of a variety of estimators of (natural or pure) direct and indirect effects (IPW, multiply robust estimation, g-computation, parametric estimation based on simulating potential mediators and outcomes, flexible parametric estimation, OLS) using a simulation design based on empirical data from Behncke, Frölich, and Lechner (2010b). Several features like the distribution of the outcome variable, the sample size, effect heterogeneity, selection into the mediator, the share of mediated, the correct or incorrect specification of econometric models, and the trimming of influential observations were varied to analyze a comprehensive set of simulation designs. Not surprisingly, we found that stronger selection into the mediator increased the RMSE (in particular under the smaller sample size) and that misspecifications in which confounders were omitted increased the bias, but decreased the variance. In contrast, trimming influential observations did not matter much for the performance of the estimators.

Our analysis did not point to a clear-cut best performing estimator for all (binary and nonbinary) outcomes and (homogeneous/heterogeneous) direct and indirect effects considered.
Concerning the direct effects, g-computation most often dominated the other methods (followed by multiply robust targeted MLE), but all estimators performed similarly well. With regard to the indirect effects, flexible parametric estimation outperformed the competitors in all scenarios when the outcome was nonbinary, but only under homogeneous effects when the outcome was binary. Under heterogeneous effects, IPW and (multiply robust) influence function-based estimation performed best and were overall the preferred choices for the indirect effects in the binary outcome case. Finally, we assessed the performance of each method when jointly estimating direct and indirect effects based on the norm of the joint RMSE matrix. For the nonbinary outcome, g-computation and flexible parametric estimation performed similarly well and dominated the remaining methods. For the binary outcome, g-computation and influence function-based estimation were ahead, albeit weighting and targeted MLE came close. Even though g-computation appeared to be the overall winner across our scenarios, the differences between estimators were often (but not always) minor. Future research may consider further estimation approaches and simulation designs that mimic empirically relevant problems different to this article to broaden the evidence on the finite sample behavior of estimators of direct and indirect effects.

\section{A. APPENDIX}

\section{A.1. Descriptive Statistics}

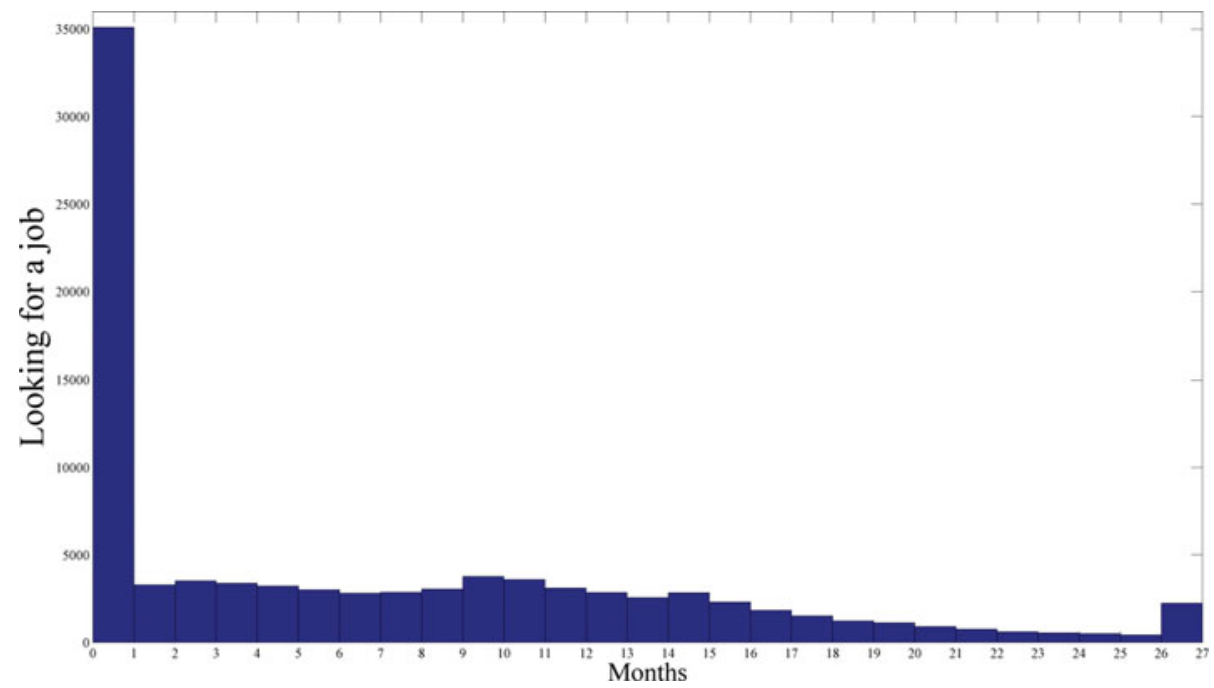

Figure A.1 Distribution of the nonbinary outcome (cumulative months jobseeking between months 10 and 36 ). 
Table A.1. Difference in observed covariates across treatment and mediator states

\begin{tabular}{|c|c|c|c|c|}
\hline Characteristics of the unemployed person & \multicolumn{2}{|c|}{ Treatment status } & \multicolumn{2}{|c|}{ Mediation status } \\
\hline Qualification: unskilled & $\begin{array}{c}0.21 \\
(0.41)\end{array}$ & $\begin{array}{c}0.22 \\
(0.42)\end{array}$ & $\begin{array}{c}0.22 \\
(0.41)\end{array}$ & $\begin{array}{c}0.17 \\
(0.37)\end{array}$ \\
\hline Qualification: skilled without degree & $\begin{array}{c}0.04 \\
(0.20)\end{array}$ & $\begin{array}{c}0.04 \\
(0.21)\end{array}$ & $\begin{array}{c}0.04 \\
(0.20)\end{array}$ & $\begin{array}{c}0.04 \\
(0.19)\end{array}$ \\
\hline Qualification: skilled with degree & $\begin{array}{c}0.59 \\
(0.49)\end{array}$ & $\begin{array}{c}0.57 \\
(0.50)\end{array}$ & $\begin{array}{c}0.57 \\
(0.49)\end{array}$ & $\begin{array}{c}0.64 \\
(0.48)\end{array}$ \\
\hline Employability rating: low & $\begin{array}{c}0.13 \\
(0.34)\end{array}$ & $\begin{array}{c}0.14 \\
(0.35)\end{array}$ & $\begin{array}{c}0.14 \\
(0.35)\end{array}$ & $\begin{array}{c}0.11 \\
(0.32)\end{array}$ \\
\hline Mother tongue other than German, French, Italian & $\begin{array}{c}0.31 \\
(0.46)\end{array}$ & $\begin{array}{c}0.32 \\
(0.47)\end{array}$ & $\begin{array}{c}0.32 \\
(0.47)\end{array}$ & $\begin{array}{c}0.24 \\
(0.43)\end{array}$ \\
\hline Number of unemployment spells in last two years & $\begin{array}{c}0.56 \\
(1.18)\end{array}$ & $\begin{array}{c}0.57 \\
(1.21)\end{array}$ & $\begin{array}{c}0.58 \\
(1.22)\end{array}$ & $\begin{array}{c}0.29 \\
(0.78)\end{array}$ \\
\hline Fraction of time employed in last two years & $\begin{array}{c}0.80 \\
(0.25)\end{array}$ & $\begin{array}{c}0.80 \\
(0.25)\end{array}$ & $\begin{array}{c}0.80 \\
(0.25)\end{array}$ & $\begin{array}{c}0.82 \\
(0.25)\end{array}$ \\
\hline \multicolumn{5}{|c|}{ Characteristics of the case worker } \\
\hline Female & $\begin{array}{c}0.42 \\
(0.49)\end{array}$ & $\begin{array}{c}0.42 \\
(0.49)\end{array}$ & $\begin{array}{c}0.42 \\
(0.49)\end{array}$ & $\begin{array}{c}0.43 \\
(0.50)\end{array}$ \\
\hline Age & $\begin{array}{c}45.1 \\
(11.96)\end{array}$ & $\begin{array}{c}43.78 \\
(11.46)\end{array}$ & $\begin{array}{c}44.45 \\
(11.77)\end{array}$ & $\begin{array}{c}44.89 \\
(11.41)\end{array}$ \\
\hline Education: tertiary track (university or polytechnic) & $\begin{array}{c}0.24 \\
(0.43)\end{array}$ & $\begin{array}{c}0.24 \\
(0.43)\end{array}$ & $\begin{array}{c}0.24 \\
(0.43)\end{array}$ & $\begin{array}{c}0.24 \\
(0.43)\end{array}$ \\
\hline Degree in vocational training for caseworkers & $\begin{array}{c}0.19 \\
(0.39)\end{array}$ & $\begin{array}{c}0.22 \\
(0.42)\end{array}$ & $\begin{array}{c}0.21 \\
(0.41)\end{array}$ & $\begin{array}{c}0.18 \\
(0.39)\end{array}$ \\
\hline Indicator for missing caseworker characteristics & $\begin{array}{c}0.04 \\
(0.19)\end{array}$ & $\begin{array}{c}0.05 \\
(0.21)\end{array}$ & $\begin{array}{c}0.04 \\
(0.20)\end{array}$ & $\begin{array}{c}0.03 \\
(0.18)\end{array}$ \\
\hline \multicolumn{5}{|c|}{ Allocation of unemployed to caseworkers } \\
\hline By industry & $\begin{array}{c}0.52 \\
(0.50)\end{array}$ & $\begin{array}{c}0.58 \\
(0.49)\end{array}$ & $\begin{array}{c}0.55 \\
(0.50)\end{array}$ & $\begin{array}{c}0.53 \\
(0.50)\end{array}$ \\
\hline By occupation & $\begin{array}{c}0.52 \\
(0.50)\end{array}$ & $\begin{array}{c}0.61 \\
(0.49)\end{array}$ & $\begin{array}{c}0.56 \\
(0.50)\end{array}$ & $\begin{array}{l}0.56 \\
(0.5)\end{array}$ \\
\hline By age & $\begin{array}{c}0.03 \\
(0.17)\end{array}$ & $\begin{array}{c}0.04 \\
(0.19)\end{array}$ & $\begin{array}{c}0.03 \\
(0.18)\end{array}$ & $\begin{array}{c}0.04 \\
(0.19)\end{array}$ \\
\hline By employability & $\begin{array}{c}0.08 \\
(0.26)\end{array}$ & $\begin{array}{c}0.07 \\
(0.25)\end{array}$ & $\begin{array}{c}0.07 \\
(0.26)\end{array}$ & $\begin{array}{c}0.06 \\
(0.25)\end{array}$ \\
\hline By region & $\begin{array}{c}0.12 \\
(0.33)\end{array}$ & $\begin{array}{c}0.12 \\
(0.32)\end{array}$ & $\begin{array}{c}0.12 \\
(0.33)\end{array}$ & $\begin{array}{c}0.09 \\
(0.29)\end{array}$ \\
\hline Other & $\begin{array}{c}0.08 \\
(0.27)\end{array}$ & $\begin{array}{c}0.08 \\
(0.27)\end{array}$ & $\begin{array}{c}0.08 \\
(0.27)\end{array}$ & $\begin{array}{c}0.08 \\
(0.27)\end{array}$ \\
\hline
\end{tabular}


Table A.1. Difference in observed covariates across treatment and mediator states (continued)

\begin{tabular}{|c|c|c|c|c|}
\hline \multirow{2}{*}{ Characteristics of the unemployed person } & \multicolumn{2}{|c|}{ Treatment status } & \multicolumn{2}{|c|}{ Mediation status } \\
\hline & Nontreated & Treated & Nonmediated & Mediated \\
\hline \multirow[t]{2}{*}{ German speaking employment office } & 0.73 & 0.77 & 0.75 & 0.70 \\
\hline & $(0.45)$ & $(0.42)$ & $(0.43)$ & $(0.46)$ \\
\hline French speaking employment office & 0.27 & 0.23 & 0.25 & 0.3 \\
\hline \multirow[t]{2}{*}{ Cantonal unemployment rate } & 3.67 & 3.71 & 3.69 & 3.66 \\
\hline & $(0.84)$ & $(0.88)$ & $(0.86)$ & $(0.84)$ \\
\hline
\end{tabular}

NOTE: Standard deviations in parentheses.

Table A.2. Probit estimates when regressing (a) $D$ on $X$ and (b) $M$ on $D$ and $X$

\begin{tabular}{|c|c|c|c|c|}
\hline$n=93,076$ & Coefficients & Std errors & Coefficients & Std errors \\
\hline Noncooperative caseworker & - & - & -0.07 & 0.02 \\
\hline French speaking employment office & 1.40 & 0.71 & 0.28 & 0.2 \\
\hline \multicolumn{5}{|c|}{ Characteristics of the case worker } \\
\hline${ }^{*}$ French & -0.02 & 0.01 & 0.00 & 0.00 \\
\hline Female & -0.04 & 0.10 & 0.00 & 0.03 \\
\hline${ }^{*}$ French & 0.07 & 0.20 & 0.06 & 0.04 \\
\hline Tenure in employment office (in years) & 0.02 & 0.02 & 0.01 & 0.00 \\
\hline *French & -0.03 & 0.03 & -0.01 & 0.01 \\
\hline Own experience of unemployment & -0.04 & 0.10 & -0.02 & 0.03 \\
\hline Education: tertiary track (university or polytechnic) & -0.20 & 0.14 & 0.03 & 0.04 \\
\hline *French & 0.31 & 0.27 & -0.10 & 0.06 \\
\hline Special vocational training of caseworker & 0.09 & 0.12 & -0.06 & 0.03 \\
\hline *French & 0.30 & 0.35 & 0.00 & 0.07 \\
\hline \multicolumn{5}{|c|}{ Allocation of unemployed to caseworkers } \\
\hline By industry & 0.14 & 0.10 & -0.03 & 0.03 \\
\hline *French & -0.07 & 0.20 & 0.00 & 0.05 \\
\hline By occupation & 0.24 & 0.10 & -0.02 & 0.03 \\
\hline *French & 0.16 & 0.20 & 0.01 & 0.05 \\
\hline By age & 0.13 & 0.23 & 0.10 & 0.05 \\
\hline *Fench & 0.10 & 0.06 & -0.14 & 0.04 \\
\hline Qualification:unskilled & 0.09 & 0.04 & -0.07 & 0.02 \\
\hline *French & -0.13 & 0.08 & -0.04 & 0.05 \\
\hline Qualification: semiskilled & 0.04 & 0.05 & 0.02 & 0.03 \\
\hline *French & -0.01 & 0.08 & -0.05 & 0.05 \\
\hline Qualification: skilled without degree & 0.02 & 0.05 & -0.05 & 0.05 \\
\hline *French & 0.19 & 0.09 & 0.03 & 0.07 \\
\hline Number of unemployment spells in last two years & 0.01 & 0.01 & -0.12 & 0.01 \\
\hline *Fench & -0.01 & 0.01 & -0.03 & 0.02 \\
\hline
\end{tabular}


Table A.2. Probit estimates when regressing (a) $D$ on $X$ and (b) $M$ on $D$ and $X$ (continued)

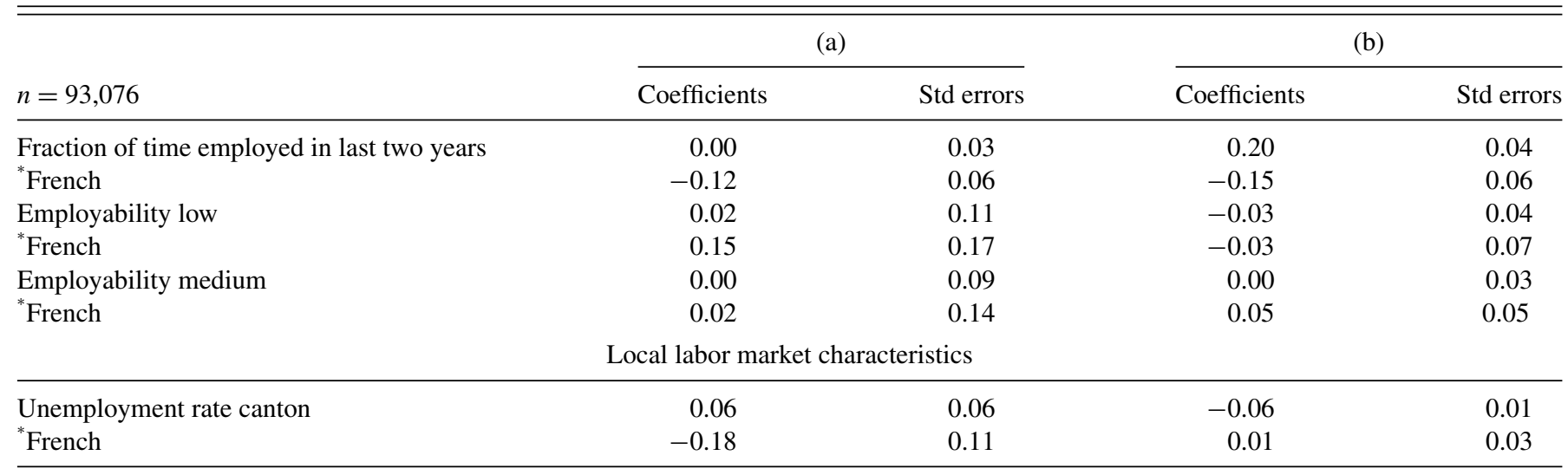

NOTE: Standard errors are clustered at caseworker level.

\section{A.2. Further Results}

Table A.3. OLS analysis of determinants of the abs. bias for the nonbinary outcome

\begin{tabular}{|c|c|c|c|c|c|c|c|c|}
\hline \multicolumn{9}{|c|}{ Direct effect } \\
\hline Constant & 0.036 & 0.016 & 0.028 & 0.024 & 0.030 & 0.024 & 0.018 & -0.008 \\
\hline Effect heterogeneity & 0.017 & 0.030 & 0.024 & 0.028 & 0.017 & 0.025 & 0.110 & 0.128 \\
\hline Misspecification & 0.056 & 0.062 & 0.051 & 0.050 & 0.051 & 0.052 & 0.053 & 0.067 \\
\hline Trimming & -0.001 & -0.003 & 0.001 & -0.004 & 0.002 & -0.002 & -0.005 & -0.002 \\
\hline TMLE/g-comp. parsimon. & & & 0.006 & 0.001 & 0.006 & -0.002 & & \\
\hline TMLE/g-comp. flexible & & & -0.000 & -0.000 & 0.000 & 0.000 & & \\
\hline DR/simulation parsimon. & & & 0.006 & 0.001 & 0.005 & -0.002 & & \\
\hline OLS flexible & & & & & & & -0.095 & -0.100 \\
\hline Constant & -0.001 & -0.000 & -0.004 & -0.013 & -0.004 & -0.008 & -0.002 & -0.003 \\
\hline Effect heterogeneity & 0.005 & 0.001 & 0.004 & 0.004 & 0.003 & 0.004 & 0.013 & 0.009 \\
\hline Strong selection & 0.019 & 0.015 & 0.023 & 0.025 & 0.019 & 0.019 & 0.028 & 0.024 \\
\hline $50 \%$ mediated & 0.004 & -0.001 & -0.002 & -0.000 & 0.000 & 0.001 & 0.009 & 0.005 \\
\hline Misspecification & 0.000 & 0.002 & 0.018 & 0.021 & 0.009 & 0.011 & -0.003 & 0.002 \\
\hline Trimming & -0.002 & 0.003 & 0.001 & 0.001 & 0.001 & 0.001 & -0.001 & 0.004 \\
\hline TMLE/g-comp. parsimon. & & & -0.001 & 0.006 & -0.001 & 0.003 & & \\
\hline TMLE/g-comp. flexible & & & -0.002 & 0.003 & 0.000 & -0.000 & & \\
\hline DR/simulation parsimon. & & & -0.007 & -0.002 & -0.001 & 0.003 & & \\
\hline OLS flexible & & & & & & & -0.008 & -0.009 \\
\hline Adjusted $R^{2}$ & 0.626 & 0.412 & 0.567 & 0.637 & 0.586 & 0.568 & 0.508 & 0.433 \\
\hline
\end{tabular}


Table A.4. OLS analysis of determinants of the abs. bias for the binary outcome

\begin{tabular}{|c|c|c|c|c|c|c|c|c|}
\hline \multicolumn{9}{|c|}{ Direct effect } \\
\hline Constant & 1.008 & 0.978 & -5.568 & 0.618 & 1.245 & 0.972 & 0.768 & 0.805 \\
\hline Effect heterogeneity & 2.194 & 2.158 & 16.748 & 5.986 & 2.180 & 2.117 & 6.105 & 5.915 \\
\hline Misspecification & 0.031 & 0.137 & 1.317 & -3.001 & -0.042 & 0.095 & 0.113 & 0.030 \\
\hline Trimming & 0.069 & 0.027 & -0.352 & -0.036 & -0.009 & 0.009 & -0.096 & -0.002 \\
\hline TMLE/g-comp. parsimon. & & & -0.112 & 0.020 & -0.102 & 0.144 & & \\
\hline TMLE/g-comp. flexible & & & 29.061 & 7.683 & -0.055 & 0.036 & & \\
\hline DR/simulation parsimon. & & & -0.112 & 0.020 & -0.181 & 0.054 & & \\
\hline OLS flexible & & & & & & & -3.819 & -3.765 \\
\hline Constant & -0.038 & -0.034 & -0.075 & -0.328 & -0.071 & -0.152 & -0.281 & -0.466 \\
\hline Effect heterogeneity & 0.031 & 0.021 & 0.447 & 0.304 & 0.090 & 0.110 & 1.051 & 1.029 \\
\hline Strong selection & 0.066 & 0.059 & 0.264 & 0.288 & 0.161 & 0.148 & 0.765 & 0.726 \\
\hline $50 \%$ mediated & 0.042 & 0.045 & 0.011 & 0.108 & 0.014 & 0.068 & 0.192 & 0.421 \\
\hline Misspecification & -0.012 & 0.006 & -0.080 & 0.080 & -0.018 & 0.033 & -0.022 & 0.205 \\
\hline Trimming & 0.029 & 0.008 & 0.020 & 0.001 & 0.015 & -0.000 & 0.098 & 0.003 \\
\hline TMLE/g-comp. parsimon. & & & 0.008 & 0.230 & 0.070 & 0.111 & & \\
\hline TMLE/g-comp. flexible & & & 0.424 & 0.343 & -0.015 & -0.004 & & \\
\hline DR/simulation parsimon. & & & -0.226 & -0.038 & 0.073 & 0.118 & & \\
\hline OLS flexible & & & & & & & -0.371 & -0.323 \\
\hline Adjusted $R^{2}$ & 0.401 & 0.506 & 0.533 & 0.406 & 0.346 & 0.376 & 0.605 & 0.569 \\
\hline
\end{tabular}

NOTE: The binary outcome has been multiplied by 100 .

Table A.5. OLS analysis of determinants of the std. dev. for the nonbinary outcome

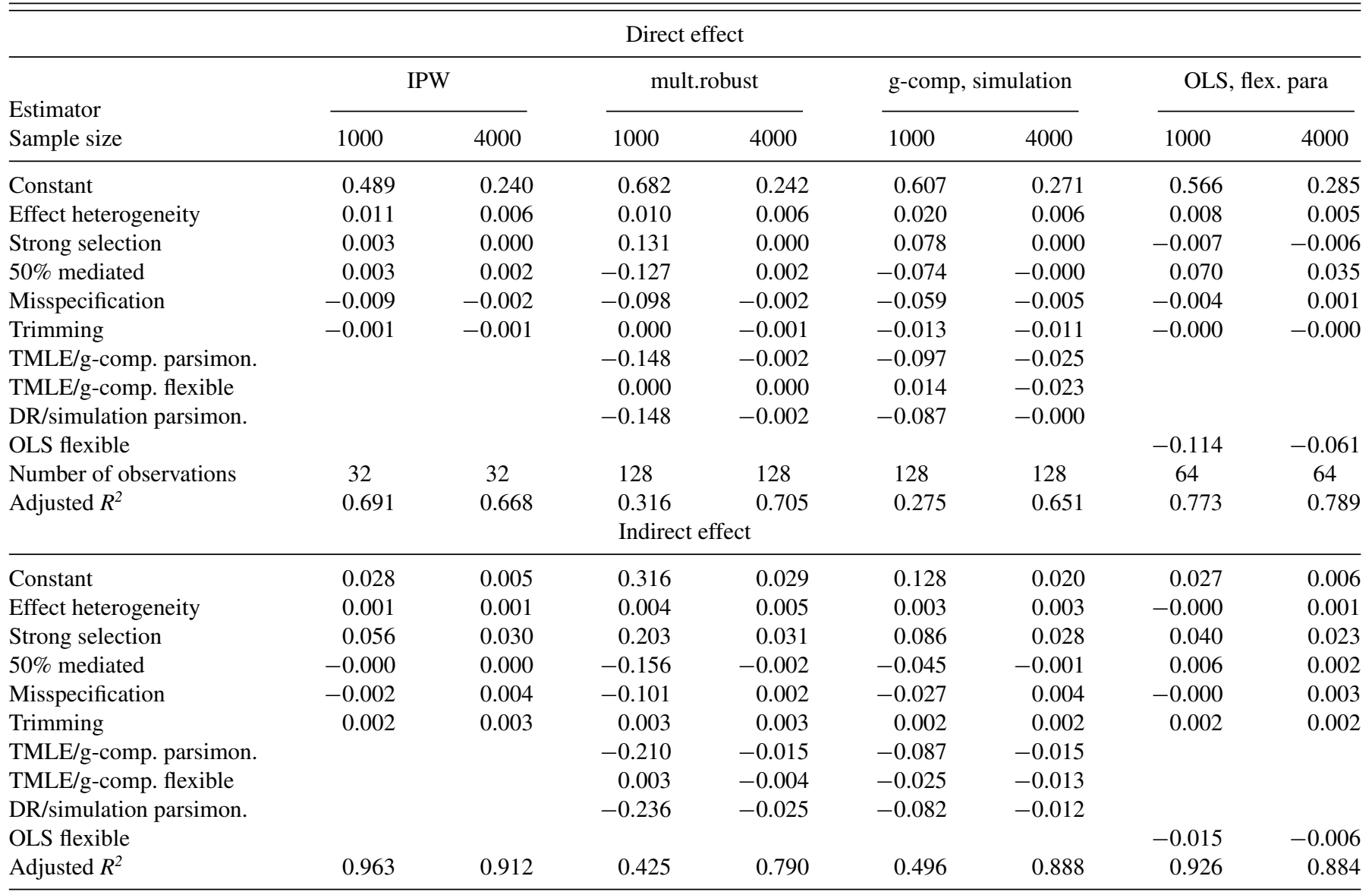


Table A.6. OLS analysis of determinants of the std. dev. for the binary outcome

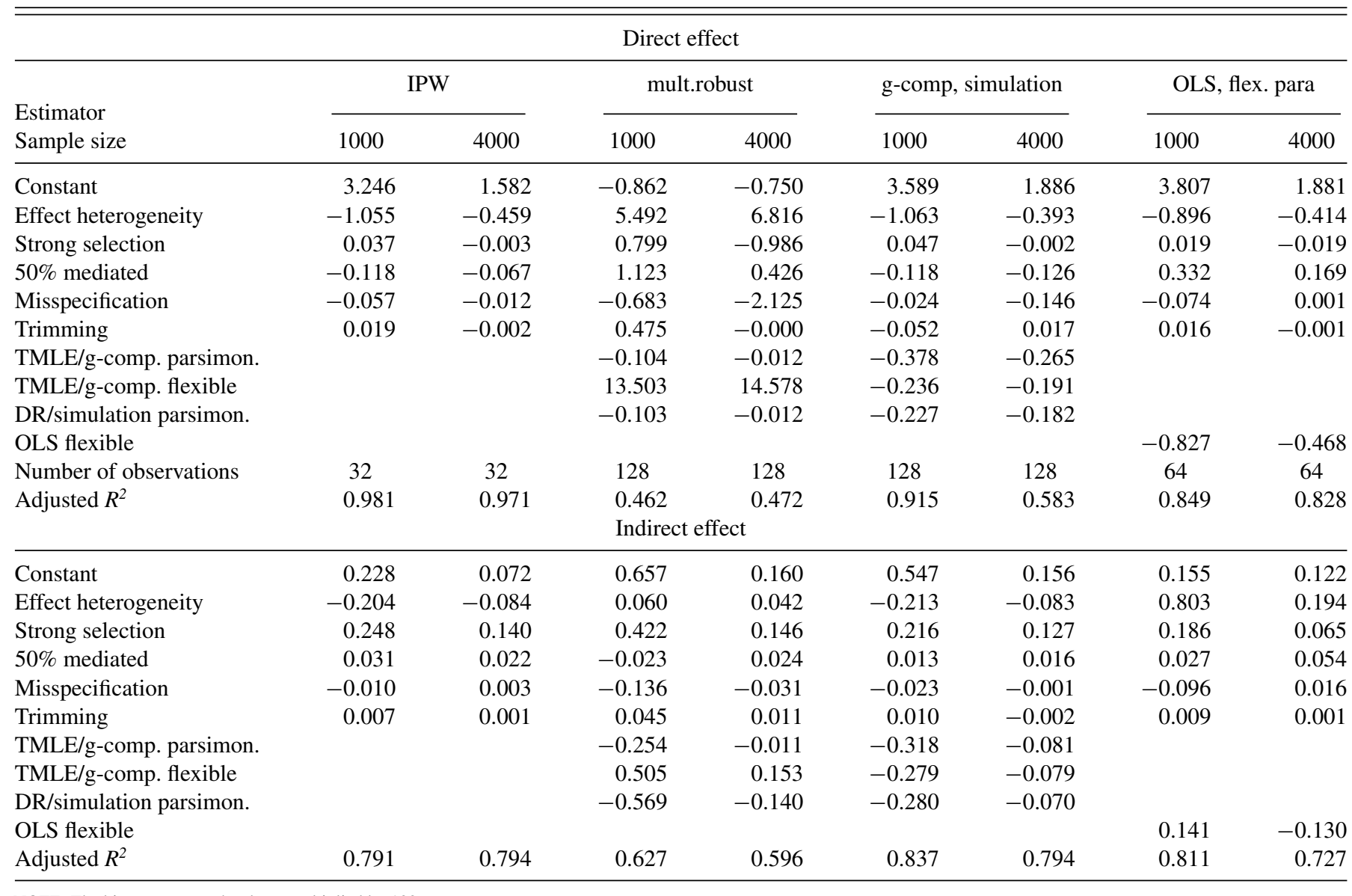

NOTE: The binary outcome has been multiplied by 100 .

Table A.7. Relative and absolute performances (direct and indirect effects jointly)

\begin{tabular}{|c|c|c|c|c|c|c|c|c|c|c|c|}
\hline nonbinary outc. & no trim. & hom. ef. & het. ef. & $n=1000$ & $n=4000$ & n. sel. & str. sel. & cor. sp. & mis. & $10 \% \mathrm{~m}$ & $50 \% \mathrm{~m}$. \\
\hline DE: g-comp. pars. IE: TMLE flex. & 0.209 & 0.203 & 0.215 & 0.246 & 0.062 & 0.207 & 0.377 & 0.348 & 0.211 & 0.368 & 0.210 \\
\hline DE: g-comp. pars. IE: influence flex. & 0.207 & 0.202 & 0.213 & 0.243 & 0.063 & 0.208 & 0.235 & 0.205 & 0.209 & 0.233 & 0.209 \\
\hline DE: g-comp. pars. IE: flex. parametric & 0.207 & 0.200 & 0.213 & 0.243 & 0.062 & 0.208 & 0.205 & 0.204 & 0.21 & 0.204 & 0.209 \\
\hline DE: flex. parametric IE: TMLE flex. & 0.208 & 0.201 & 0.214 & 0.244 & 0.064 & 0.209 & 0.373 & 0.346 & 0.208 & 0.366 & 0.210 \\
\hline DE: flex. parametric IE: influence flex. & 0.207 & 0.201 & 0.214 & 0.243 & 0.064 & 0.211 & 0.233 & 0.206 & 0.209 & 0.232 & 0.209 \\
\hline DE: flex. parametric IE: g-comp. pars. & 0.207 & 0.201 & 0.214 & 0.243 & 0.064 & 0.21 & 0.205 & 0.206 & 0.209 & 0.205 & 0.210 \\
\hline Both: IPW & 0.215 & 0.208 & 0.222 & 0.253 & 0.064 & 0.214 & 0.216 & 0.215 & 0.215 & 0.212 & 0.218 \\
\hline Both: TMLE pars. & 0.215 & 0.208 & 0.222 & 0.253 & 0.064 & 0.214 & 0.216 & 0.214 & 0.215 & 0.213 & 0.217 \\
\hline Both: influence pars. & 0.214 & 0.206 & 0.221 & 0.251 & 0.063 & 0.213 & 0.214 & 0.213 & 0.214 & 0.211 & 0.216 \\
\hline Both: g-comp. pars. & 0.208 & 0.201 & 0.215 & 0.244 & 0.062 & 0.208 & 0.208 & 0.205 & 0.211 & 0.206 & 0.210 \\
\hline Both: flex. parametric & 0.207 & 0.200 & 0.214 & 0.243 & 0.0630 & 0.209 & 0.205 & 0.206 & 0.209 & 0.204 & 0.210 \\
\hline binary outc. & no trim. & hom. ef. & het. ef. & $n=1000$ & $n=4000$ & n. sel. & str. sel. & cor. sp. & mis. & $10 \% \mathrm{~m}$ & $50 \% \mathrm{~m}$ \\
\hline DE: TMLE pars. IE: influence pars. & 0.101 & 0.087 & 0.116 & 0.113 & 0.057 & 0.123 & & & 0.100 & & 0.064 \\
\hline DE: TMLE pars. IE: flex. parametric & 0.102 & 0.086 & 0.119 & 0.113 & 0.057 & 0.123 & 0.081 & 0.102 & 0.102 & 0.142 & 0.064 \\
\hline DE: g-comp. pars. IE: TMLE pars. & 0.102 & 0.086 & 0.118 & 0.113 & 0.059 & 0.124 & 0.080 & 0.102 & 0.101 & 0.141 & 0.065 \\
\hline DE: g-comp. pars. IE: influence pars. & 0.101 & 0.085 & 0.116 & 0.111 & 0.059 & 0.123 & 0.078 & 0.103 & 0.098 & 0.136 & 0.065 \\
\hline DE: g-comp. pars. IE: flex. parametric & 0.100 & 0.085 & 0.116 & 0.111 & 0.061 & 0.124 & 0.076 & 0.104 & 0.099 & 0.138 & 0.067 \\
\hline Both: IPW & 0.104 & 0.087 & 0.121 & 0.115 & 0.058 & 0.126 & 0.082 & 0.105 & 0.102 & 0.143 & 0.065 \\
\hline Both: TMLE pars. & 0.104 & 0.088 & 0.120 & 0.115 & 0.058 & 0.123 & 0.084 & 0.104 & 0.104 & 0.144 & 0.063 \\
\hline Both: influence pars. & 0.101 & 0.087 & 0.116 & 0.113 & 0.057 & 0.123 & 0.080 & 0.103 & 0.100 & 0.139 & 0.064 \\
\hline Both: g-comp. pars. & 0.101 & 0.085 & 0.116 & 0.111 & 0.060 & 0.123 & 0.078 & 0.103 & 0.098 & 0.137 & 0.066 \\
\hline Both: flex. parametric & 0.113 & 0.084 & 0.142 & 0.125 & 0.067 & 0.143 & 0.083 & 0.123 & 0.103 & 0.150 & 0.076 \\
\hline
\end{tabular}

NOTE: DE = direct effect; IE = indirect effect. Only simulations without trimming are considered. 


\section{ACKNOWLEDGMENTS}

Michael Lechner is a Research Fellow of CEPR and PSI, London, CES-Ifo, Munich, IAB, Nuremberg, and IZA, Bonn. We have benefited from comments and advice by Kosuke Imai, Eric Tchetgen Tchetgen, Mark van der Laan, Teppei Yamamoto, Wen Zheng, an associate editor, and three anonymous referees. The usual disclaimer applies.

\section{FUNDING}

Michael Lechner and Giovanni Mellace gratefully acknowledge financial support from the Swiss National Science Foundation grant number 100018 137769.

\section{[Received June 2014. Revised January 2015.]}

\section{REFERENCES}

Advani, A., and Sloczyński, T. (2013), "Mostly Harmless Simulations? On the Internal Validity of Empirical Monte Carlo Studies," IZA Discussion Paper No. 7874, Bonn: Institute for the Study of Labor (IZA). [140]

Albert, J. M. (2008), "Mediation Analysis via Potential Outcomes Models," Statistics in Medicine, 27, 1282-1304. [141]

Albert, J. M., and Nelson, S. (2011), "Generalized Causal Mediation Analysis," Biometrics, 67, 1028-1038. [139]

Avin, C., Shpitser, I., and Pearl, J. (2005), "Identifiability of Path-Specific Effects," in IJCAI-05, Proceedings of the Nineteenth International Joint Conference on Artificial Intelligence, Edinburgh, UK, pp. 357363. [139]

Baron, R. M., and Kenny, D. A. (1986), "The Moderator-Mediator Variable Distinction in Social Psychological Research: Conceptual, Strategic, and Statistical Considerations," Journal of Personality and Social Psychology, 51, 1173-1182. [140,142]

Behncke, S., Frölich, M., and Lechner, M. (2010a), "A Caseworker Like Me: Does The Similarity Between The Unemployed and Their Caseworkers Increase Job Placements?" Economic Journal, 120, 1430-1459. [145]

(2010b), "Unemployed and Their Caseworkers: Should They be Friends or Foes?" Journal of the Royal Statistical Society, Series A, 173, 67-92. [145,146,147,154]

Busso, M., DiNardo, J., and McCrary, J. (in press, a), "Finite Sample Properties of Semiparametric Estimators of Average Treatment Effects," Journal of Business and Economic Statistics. [140,144,148]

_ (in press, b), "New Evidence on the Finite Sample Properties of Propensity Score Matching and Reweighting Estimators," Review of Economics and Statistics. $[140,144]$

Flores, C. A., and Flores-Lagunes, A. (2009), "Identification and Estimation of Causal Mechanisms and Net Effects of a Treatment Under Unconfoundedness," IZA Dicussion Paper No. 4237, Bonn: Institute for the Study of Labor (IZA). [140,141]

Frölich, M. (2004), "Finite Sample Properties of Propensity-Score Matching and Weighting Estimators," Review of Economics and Statistics, 86, 77-90. [140]

Gelman, A., and Imbens, G. (2013), "Why Ask Why? Forward Causal Inference and Reverse Causal Questions," NBER Working Paper No. 19614, Cambridge, MA: National Bureau of Economic Research. [139]

Gerfin, M., and Lechner, M. (2002), "Microeconometric Evaluation of the Active Labour Market Policy in Switzerland," Economic Journal, 112, 854-893. [147]

Hill, W. J., and Hunter, W. G. (1966), "A Review of Response Surface Methodology: A Literature Survey," Technometrics, 8, 571-590. [149]

Horvitz, D. G., and Thompson, D. J. (1952), "A Generalization of Sampling Without Replacement From a Finite Universe," Journal of the American Statistical Association, 47, 663-685. [140,144]

Huber, M. (2014), "Identifying Causal Mechanisms (Primarily) Based on Inverse Probability Weighting," Journal of Applied Econometrics, 29, 920 943. [139,140,141,142,144]

Huber, M., Lechner, M., and Mellace, G. (2014), "Why Do Tougher Caseworkers Increase Employment? The Role of Programme Assignment as a Causal Mechanism," Department of Economics Discussion Paper No. 2014-14, University of St. Gallen. [140,146]
Huber, M., Lechner, M., and Wunsch, C. (2013), "The Performance of Estimators Based on the Propensity Score," Journal of Econometrics, 175, 1-21. $[140,144,145,148]$

Imai, K., Keele, L., and Tingley, D. (2010a), "A General Approach to Causal Mediation Analysis," Psychological Methods, 15, 309-334. [142,143]

Imai, K., Keele, L., and Yamamoto, T. (2010b), "Identification, Inference and Sensitivity Analysis for Causal Mediation Effects," Statistical Science, 25, 51-71. [140,141,142]

Imai, K., and Yamamoto, T. (2013), "Identification and Sensitivity Analysis for Multiple Causal Mechanisms: Revisiting Evidence From Framing Experiments," Political Analysis, 21, 141-171. [139]

Imbens, G. W. (2004), "Nonparametric Estimation of Average Treatment Effects Under Exogeneity: A Review," Review of Economics and Statistics, 86, 4 29. [141]

Kang, J. D. Y., and Schafer, J. L. (2007), "Demystifying Double Robustness: A Comparison of Alternative Strategies for Estimating a Population Mean From Incomplete Data," Statistical Science, 22, 523-539. [144]

Khan, S., and Tamer, E. (2010), "Irregular Identification, Support Conditions, and Inverse Weight Estimation," Econometrica, 78, 2021-2042. $[144,148]$

Khuri, A. I., and Mukhopadhyay, S. (2010), "Response Surface Methodology," Wiley Interdisciplinary Reviews: Computational Statistics, 2, 128-149. [149]

LaLonde, R. (1986), "Evaluating the Econometric Evaluations of Training Programs With Experimental Data," American Economic Review, 76, 604-620. [140]

Lechner, M., and Wunsch, C. (2013), "Sensitivity of Matching-Based Program Evaluations to the Availability of Control Variables," Labour Economics, 21, 111-121. [147]

Lunceford, J. K., and Davidian, M. (2004), "Stratification and Weighting via the Propensity Score in Estimation of Causal Treatment Effects: A Comparative Study," Statistics in Medicine, 23, 2937-2960. [140]

Myers, R. H., Khuri, A. I., and Carter, W. H., Jr, (1989), "Response Surface Methodology: 1966-1988," Technometrics, 31, 137-157. [149]

Nargelkerke, N. J. D. (1991), "A Note on a General Definition of the Coefficient of Determination," Biometrika, 78, 691-692. [148]

Newey, W. K. (1984), "A Method of Moments Interpretation of Sequential Estimators," Economics Letters, 14, 201-206. [144]

Pearl, J. (2001), "Direct and Indirect Effects," in Proceedings of the Seventeenth Conference on Uncertainty in Artificial Intelligence, San Francisco, CA: Morgan Kaufman, pp. 411-420. [140,141,142]

Robins, J. M. (1986), "A New Approach to Causal Inference in Mortality Studies With Sustained Exposure Periods: Application to Control of the Healthy Worker Survivor Effect," Mathematical Modelling, 7, 1393-1512. $[140,142,143]$

(2003), "Semantics of Causal DAG Models and the Identification of Direct and Indirect Effects," in Highly Structured Stochastic Systems, eds. P. Green, N. Hjort, and S. Richardson, Oxford: Oxford University Press, pp. 70-81. $[139,141,142]$

Robins, J. M., and Greenland, S. (1992), "Identifiability and Exchangeability for Direct and Indirect Effects," Epidemiology, 3, 143-155. [141]

Rubin, D. B. (1974), "Estimating Causal Effects of Treatments in Randomized and Nonrandomized Studies," Journal of Educational Psychology, 66, 688701. [141]

- (2004), "Direct and Indirect Causal Effects via Potential Outcomes," Scandinavian Journal of Statistics, 31, 161-170. [141]

Tchetgen Tchetgen, E. J., and Shpitser, I. (2012), "Semiparametric Theory for Causal Mediation Analysis: Efficiency Bounds, Multiple Robustness, and Sensitivity Analysis," The Annals of Statistics, 40, 1816-1845. $[140,141,142,144,145]$

Ten Have, T. R., Joffe, M. M., Lynch, K. G., Brown, G. K., Maisto, S. A., and Beck, A. T. (2007), "Causal Mediation Analyses With Rank Preserving Models," Biometrics, 63, 926-934. [141]

Tingley, D., Yamamoto, T., Hirose, K., Imai, K., and Keele, L. (2014), "Mediation: R Package for Causal Mediation Analysis," Journal of Statistical Software, 59, 1-38. [140,142,143,144]

van der Laan, M., and Rubin, D. (2006), “Targeted Maximum Likelihood Learning," International Journal of Biostatistics, 2, Article 11. [145]

Waernbaum, I. (2012), "Model Misspecification and Robustness in Causal Inference: Comparing Matching With Doubly Robust Estimation,” Statistics in Medicine, 31, 1572-1581. [144]

Zhao, Z. (2004), "Using Matching to Estimate Treatment Effects: Data Requirements, Matching Metrics, and Monte Carlo Evidence," Review of Economics and Statistics, 86, 91-107. [140]

Zheng, W., and van der Laan, M. J. (2012), "Targeted Maximum Likelihood Estimation of Natural Direct Effects," International Journal of Biostatistics, 8, 1-40. [140,142,143,144,145] 